\title{
CÉDRIC BONNAFÉ
}

\section{Opérateur de torsion dans $S L_{n}(q)$ et $S U_{n}(q)$}

Bulletin de la S. M. F., tome 128, no 3 (2000), p. 309-345

<http://www.numdam.org/item?id=BSMF_2000_128_3_309_0>

(C) Bulletin de la S. M. F., 2000, tous droits réservés.

L'accès aux archives de la revue «Bulletin de la S. M. F. » (http: //smf.emath.fr/Publications/Bulletin/Presentation.html) implique l'accord avec les conditions générales d'utilisation (http://www.numdam.org/ conditions). Toute utilisation commerciale ou impression systématique est constitutive d'une infraction pénale. Toute copie ou impression de ce fichier doit contenir la présente mention de copyright.

\section{Numdam}


Bull. Soc. math. France,

128, 2000, p. 309-345.

\title{
OPÉRATEUR DE TORSION DANS $\mathrm{SL}_{n}(q)$ ET $\mathrm{SU}_{n}(q)$
}

\author{
PAR CÉdRIC BONNAFÉ $(*)$
}

\begin{abstract}
RÉSUMÉ. - Nous calculons l'opérateur de torsion dans tous les groupes réductifs finis de type $A$ lorsque l'endomorphisme de Frobenius agit trivialement sur le le groupe des composantes du centre. Le résultat s'applique aussi bien au groupe $\operatorname{SL}_{n}(q)$ qu'à la forme tordue $\mathrm{SU}_{n}(q)$.

ABstract. - TWisting operators in $\operatorname{SL}_{n}(q)$ AND $\mathrm{SU}_{n}(q)$. - We compute the twisting operator in every finite reductive group of type $A$, provided that the Frobenius endomorphism acts trivially on the group of components of the center. Our result applies as well in the group $\mathrm{SL}_{n}(q)$ as in the twisted form $\mathrm{SU}_{n}(q)$.
\end{abstract}

Soit $\boldsymbol{G}$ un groupe réductif défini sur un corps à $q$ éléments $\mathbb{F}_{q}$ de caractéristique $p$ et soit $F: \boldsymbol{G} \rightarrow \boldsymbol{G}$ l'endomorphisme de Frobenius correspondant. Dans le but de paramétrer les caractères (ordinaires) du groupe fini $\boldsymbol{G}^{F}$, G. Lusztig a introduit des combinaisons linéaires particulières (et explicites) des caractères irréductibles de $\boldsymbol{G}^{F}$, appelés depuis caractères fantômes (ou almost characters en anglais). Ces caractères fantômes forment une base orthonormale de l'espace $\operatorname{Cent}\left(\boldsymbol{G}^{F}\right)$ des fonctions centrales sur $\boldsymbol{G}^{F}$ et calculer la table de caractères de $\boldsymbol{G}^{F}$ se ramène au calcul des valeurs des caractères fantômes en les éléments de $\boldsymbol{G}^{F}$. Les calculs sur des petits exemples montrent que la table des caractères fantômes a une structure beaucoup plus simple que la table des caractères ordinaires. Dans une série d'articles ( $c f$. [L2] et [L3]), Lusztig a élaboré la théorie des faisceaux-caractères pour essayer de comprendre ce phénomène. Une des réalisations de cette théorie est la construction d'une nouvelle base orthonormale de l'espace vectoriel $\operatorname{Cent}\left(\boldsymbol{G}^{F}\right)$, dont les éléments, appelés fonctions

(*) Texte reçu le 19 janvier 1999, accepté le 25 mai 1999.

C. BonnafÉ, Département de Mathématiques, Université de Franche-Comté, 16 route de Gray, 25000 Besançon. Cet article a été écrit alors que l'auteur était instructeur à l'Université de Chicago (Illinois, USA). Email : bonnafe@math.univ-fcomte.fr.

Classification mathématique par matières : 20 G 05, 20 G04.

Mots clés : groupes réductifs, corps finis, caractères. 
caractéristiques de faisceaux-caractères, sont paramétrés par le même ensemble que celui qui paramètre les caractères fantômes. Lusztig a conjecturé que ces deux bases coïncident, à multiplication près par certaines racines de l'unité, et a donné un algorithme permettant de calculer les fonctions caractéristiques des faisceaux-caractères.

La preuve de cette conjecture en général constituerait une étape importante dans la connaissance de la table de caractères de $\boldsymbol{G}^{F}$. Un progrès considérable a été fait par T. Shoji qui a prouvé cette conjecture lorsque le centre de $\boldsymbol{G}$ est connexe en supposant seulement que $p$ est presque bon ( $f f$. [Sh2]) : il a même calculé explicitement les racines de l'unité invoquées ci-dessus.

Une des conséquences de la conjecture de Lusztig est que les caractères fantômes sont des vecteurs propres pour l'opérateur de torsion $\operatorname{Sh}_{F / F}^{G}(c f . \S 2.1$ pour la définition de l'opérateur de torsion $\left.\operatorname{Sh}_{F / F}^{G}: \operatorname{Cent}\left(\boldsymbol{G}^{F}\right) \rightarrow \operatorname{Cent}\left(\boldsymbol{G}^{F}\right)\right)$. Dans [A], T. Asai a étudié l'opérateur de torsion lorsque $\boldsymbol{G}$ est un sous-groupe de Levi rationnel d'un sous-groupe parabolique non nécessairement rationnel du groupe spécial linéaire $\mathrm{SL}_{n}$ muni de son endomorphisme de Frobenius déployé naturel. Cependant, le résultat obtenu [A, th. 4.5.2], à savoir le calcul explicite de l'opérateur de torsion, ne s'appliquait que lorsque $q \equiv 1 \bmod (\operatorname{ppcm}(1,2, \ldots, n))$ et malheureusement, la preuve d'Asai était fausse. Dans cet article, nous reprenons quelques idées d'Asai et donnons une preuve (que l'on espère correcte) de ce résultat dans le cas où $q \equiv 1 \bmod n$. Mieux, nous généralisons ce résultat au cas de tous les groupes de type $A$ (c'est-à-dire si toutes les composantes irréductibles du système de racines de $\boldsymbol{G}$ sont de type $A$ ). Il est à noter que la définition de groupe de type $A$ ci-dessus ne fait pas intervenir la structure rationnelle de $\boldsymbol{G}$. En particulier, le résultat que l'on obtient s'applique aussi bien au groupe spécial linéaire qu'au groupe spécial unitaire.

Plus précisément, si $\boldsymbol{G}$ est de type $A$, nous montrons essentiellement deux résultats sur l'opérateur de torsion. Le premier est que l'opérateur de torsion stabilise l'espace engendré par les caractères irréductibles de $\boldsymbol{G}^{F}$ appartenant à une même série de Lusztig géométrique, ceci sans aucune hypothèse sur $q$ ( $c f$. corollaire 4.3.5). Le deuxième est le calcul explicite de l'opérateur de torsion lorsque $F$ agit trivialement sur le groupe des composantes du centre de $\boldsymbol{G}$ (si $\boldsymbol{G}^{F}=\mathrm{SL}_{n}\left(\mathbb{F}_{q}\right)$, cette hypothèse est équivalente à $q \equiv 1 \bmod n$ ) : nous prouvons qu'alors les caractères fantômes sont des vecteurs propres pour $\mathrm{Sh}_{F / F}^{G}$ et nous calculons explicitement les valeurs propres associées ( $c f$. théorème 5.5.4). Nous utilisons pour cela deux ingrédients essentiels. Le premier est la notion de fonction absolument cuspidale ( $c f . \S 4.2$ pour la définition) : l'espace des fonctions absolument cuspidales a été déterminé dans les groupes de type $A$ dans [B2, cor. 6.2.2] et, à partir de ce résultat, il est possible de déduire que l'opérateur de torsion stabilise l'espace engendré par les caractères irréductibles de $\boldsymbol{G}^{F}$ appartenant à une même série de Lusztig géométrique. Le deuxième est

TOME $128-2000-\mathrm{N}^{\circ} 3$ 
la notion de caractère de Gel'fand-Graev généralisé. Ces caractères permettent, dans les groupes de type $A$, de paramétrer les caractères irréductibles de $G^{F}$. De plus, il est facile de calculer leur image par l'opérateur de torsion ( $c f$. prop. 2.2.5). Une récurrence portant sur les classes unipotentes de $\boldsymbol{G}$ permet alors de conclure. Cet argument n'est pas nouveau. Il est à l'œuvre dans l'article de Shoji [Sh3] dans lequel est calculée la descente de Shintani $\operatorname{Sh}_{F^{m} / F}^{\boldsymbol{G}}$ dans le groupe spécial linéaire lorsque $m$ est suffisamment divisible. Ici, nous nous intéressons au cas extrême opposé, c'est-à-dire $m=1$, et reprenons quelques idées de Shoji. Le cas $m=1$ étant plutôt plus facile, nous parvenons à mener l'argument jusqu'au bout, même dans le cas du groupe spécial unitaire.

Nous concluons cet article en montrant que, toujours sous l'hypothèse que $\boldsymbol{G}$ est de type $A$, les fonctions caractéristiques de faisceaux-caractères cuspidaux sont égales, à une racine de l'unité près, aux caractères fantômes absolument cuspidaux correspondants : c'est une première étape dans le but de prouver la conjecture de Lusztig pour ce type de groupes.

Cet article est organisé comme suit. Dans les trois premières parties, aucune hypothèse n'est faite sur le groupe $\boldsymbol{G}$. Dans la première, nous nous intéressons au groupe des composantes du centre de $\boldsymbol{G}$. Il n'est (presque) pas exagéré de dire que, dans le cas des groupes de type $A$, toutes les difficultés ont une interprétation en termes de ce groupe abélien fini. Dans la deuxième, nous rappelons la définition de l'opérateur de torsion $\mathrm{Sh}_{F / F}^{G}$ et calculons son action sur des caractères obtenus par induction à partir du radical unipotent d'un sous-groupe parabolique rationnel de $\boldsymbol{G}$. Dans la troisième, nous rappelons quelques propriétés des caractères de Gel'fand-Graev ordinaires, qui sont des cas particuliers de caractères mentionnés ci-dessus. Dans les trois dernières parties, nous nous intéressons aux groupes de type $A$. Le but de la quatrième partie est de montrer que l'opérateur de torsion stabilise l'espace vectoriel engendré par les caractères irréductibles de $\boldsymbol{G}^{F}$ appartenant à une série de Lusztig géométrique ( $c f$. corollaire 4.3.5). La cinquième partie est consacrée à l'énoncé et à la preuve du théorème principal de cette article ( $c f$. théorème 5.5.4), à savoir la description de l'opérateur de torsion. Dans la dernière partie, nous nous intéressons aux faisceaux-caractères cuspidaux $F$-stables sur le groupe $\boldsymbol{G}$.

Ce n'est qu'au cours de la preuve du théorème 5.5.4 que nous avons besoin de supposer que $F$ agit trivialement sur le groupe des composantes du centre de $\boldsymbol{G}$. Tous les autres résultats de cet article sont affranchis de cette hypothèse.

Remerciements. - L'auteur tient à remercier particulièrement Jean Michel pour avoir été à l'origine de ce travail, pour en avoir suivi attentivement l'évolution, et pour avoir lu scrupuleusement une première version de cet article. 


\section{Notations}

0.1. - Soit $p$ un nombre premier et soit $\mathbb{F}$ une clôture algébrique du corps fini à $p$ éléments $\mathbb{F}_{p}$. On fixe une puissance $q$ de $p$ et on notera $\mathbb{F}_{q}$ le sous-corps de $\mathbb{F}$ à $q$ éléments. Toutes les variétés et tous les groupes algébriques seront considérés sur $\mathbb{F}$.

On se fixe d'autre part un nombre premier $\ell$ différent de $p$ et on notera $\overline{\mathbb{Q}}_{\ell}$ une clôture algébrique du corps $\ell$-adique $\mathbb{Q}_{\ell}$. On choisit un automorphisme de corps $\overline{\mathbb{Q}}_{\ell} \rightarrow \overline{\mathbb{Q}}_{\ell}, x \mapsto \bar{x}$ tel que $\bar{\zeta}=\zeta^{-1}$ pour toute racine de l'unité $\zeta$ dans $\overline{\mathbb{Q}}_{\ell}$.

0.2. - Si $H$ est un groupe fini, on notera Irr $H$ l'ensemble des caractères irréductibles de $H$ (sur $\left.\overline{\mathbb{Q}}_{\ell}\right)$ et $\operatorname{Cent}(H)$ le $\overline{\mathbb{Q}}_{\ell}$-espace vectoriel des fonctions centrales $H \rightarrow \overline{\mathbb{Q}}_{\ell}$. Soit $H^{\wedge}$ le groupe abélien des caractères linéaires $H \rightarrow \overline{\mathbb{Q}}_{\ell}{ }^{\times}$(si $H$ est abélien, on a $H^{\wedge}=\operatorname{Irr} H$ ). On appellera $H$-module un $\overline{\mathbb{Q}}_{\ell} H$-module à gauche de dimension finie. Si $K$ est un autre groupe fini, on appellera $H$-module- $K$ un $\overline{\mathbb{Q}}_{\ell}$-espace vectoriel de dimension finie muni d'une structure de bimodule, $H$ agissant à gauche et $K$ à droite. Si $\varphi: H \rightarrow K$ est un morphisme de groupes, on notera $\widehat{\varphi}: K^{\wedge} \rightarrow H^{\wedge}$ le morphisme de groupes abéliens induit par $\varphi$. Si $\eta$ et $\eta^{\prime}$ sont deux fonctions centrales sur $H$, on notera

$$
\left\langle\eta, \eta^{\prime}\right\rangle_{H}=\frac{1}{|H|} \sum_{h \in H} \eta(h) \overline{\eta^{\prime}(h)}
$$

Alors $\langle,\rangle_{H}$ est un produit scalaire sur Cent $(H)$ pour lequel Irr $H$ est une base orthonormale. L'ensemble des classes de conjugaison de $H$ sera noté $\operatorname{Conj}(H)$. Si $g \in H$, on notera $[g]$ ou $[g]_{H}$ sa classe de conjugaison dans $H$.

Si $H$ est de plus abélien et si $\varphi: H \rightarrow H$ est un automorphisme du groupe $H$, on posera :

$$
\begin{aligned}
\mathcal{M}(H, \varphi) & =H^{1}(\varphi, H) \times\left(H^{\varphi}\right)^{\wedge}, \\
\mathcal{M}^{\vee}(H, \varphi) & =H^{\varphi} \times H^{1}(\varphi, H)^{\wedge},
\end{aligned}
$$

où $H^{1}(\varphi, H)=H /\left\{\varphi(h) h^{-1} \mid h \in H\right\}$ est le premier groupe de cohomologie du groupe cyclique $\langle\varphi\rangle$ à coefficients dans $H$. Si $(\alpha, \xi) \in \mathcal{M}(H, \varphi)$ et $(a, \zeta) \in$ $\mathcal{M}^{\vee}(H, \varphi)$, on pose :

$$
\{(\alpha, \xi),(a, \zeta)\}=\frac{\zeta(\alpha) \xi(a)}{\left|H^{\varphi}\right|} .
$$

Remarquons que $\left|H^{\varphi}\right|=\left|H^{1}(\varphi, H)\right|$.

0.3. - Si $\boldsymbol{H}$ est un groupe algébrique, on notera $\boldsymbol{H}^{0}$ sa composante neutre. Si $h \in \boldsymbol{H}$, on notera $(h)$ ou $(h)_{\boldsymbol{H}}$ la classe de conjugaison de $h$ sous $\boldsymbol{H}$ et $C_{\boldsymbol{H}}^{0}(h)$ la composante neutre de son centralisateur dans $\boldsymbol{H}$. On posera 
$A_{\boldsymbol{H}}(h)=C_{\boldsymbol{H}}(h) / C_{\boldsymbol{H}}^{0}(h)$. Si $\boldsymbol{H}$ est de plus défini sur $\mathbb{F}_{q}$ et si $F: \boldsymbol{H} \rightarrow \boldsymbol{H}$ désigne l'endomorphisme de Frobenius correspondant, on notera

$$
\varepsilon_{\boldsymbol{H}}=(-1)^{\mathbb{F}_{q} \text {-rang de } \boldsymbol{H}} \quad \text { et } \quad \eta_{\boldsymbol{H}}=(-1)^{\mathbb{F}_{q} \text {-rang semi-simple de } \boldsymbol{H}} \text {. }
$$

D'autre part, on notera $H^{1}(F, \boldsymbol{H})$ l'ensemble des classes de $F$-conjugaison de $\boldsymbol{H}$ (on rappelle que deux éléments $h$ et $h^{\prime}$ de $\boldsymbol{H}$ sont dits $F$-conjugués si il existe $x \in \boldsymbol{H}$ tel que $\left.h^{\prime}=x^{-1} h F(x)\right)$. Le théorème de Lang montre que $H^{1}(F, \boldsymbol{H})=H^{1}\left(F, \boldsymbol{H} / \boldsymbol{H}^{0}\right)$. Pour finir, on notera $\boldsymbol{Z}(\boldsymbol{H})$ et $\boldsymbol{D}(\boldsymbol{H})$ le centre et le groupe dérivé de $\boldsymbol{H}$ respectivement.

0.4. - Dans toute la suite, on se fixe un groupe algébrique réductif connexe $\boldsymbol{G}$, défini sur $\mathbb{F}_{q}$. On notera $F: \boldsymbol{G} \rightarrow \boldsymbol{G}$ l'endomorphisme de Frobenius correspondant. On notera $\boldsymbol{Z}$ le centre de $\boldsymbol{G}$. D'après [DL, 1.21], il existe un groupe réductif connexe $\widetilde{\boldsymbol{G}}$, défini sur $\mathbb{F}_{q}$ (on notera encore $F: \widetilde{\boldsymbol{G}} \rightarrow \widetilde{\boldsymbol{G}}$ l'endomorphisme de Frobenius correspondant à cette $\mathbb{F}_{q}$-structure), contenant $\boldsymbol{G}$ comme sous-groupe fermé $F$-stable et vérifiant les propriétés suivantes :

1) $\boldsymbol{G}$ contient le groupe dérivé de $\widetilde{\boldsymbol{G}}$,

2) Le centre $\widetilde{\boldsymbol{Z}}$ de $\widetilde{\boldsymbol{G}}$ est connexe.

Il résulte des propriétés 1) et 2) que $\boldsymbol{G}$ est un sous-groupe distingué de $\widetilde{\boldsymbol{G}}$, que $\widetilde{\boldsymbol{G}} / \boldsymbol{G}$ est abélien, que $\boldsymbol{Z}=\boldsymbol{G} \cap \widetilde{\boldsymbol{Z}}$ et que $\widetilde{\boldsymbol{G}}=\boldsymbol{G} \cdot \widetilde{\boldsymbol{Z}}$.

Si $z \in \boldsymbol{Z}^{F}$ et si $\gamma \in \operatorname{Cent}\left(\boldsymbol{G}^{F}\right)$, on posera

$$
\begin{aligned}
t_{z}^{\boldsymbol{G}} \gamma: \boldsymbol{G}^{F} & \longrightarrow \overline{\mathbb{Q}}_{\ell}, \\
g & \longmapsto \gamma(z g) .
\end{aligned}
$$

Alors $t_{z}^{G} \gamma$ est une fonction centrale sur $\boldsymbol{G}^{F}$ et l'application $t_{z}^{\boldsymbol{G}}: \operatorname{Cent}\left(\boldsymbol{G}^{F}\right) \rightarrow$ $\operatorname{Cent}\left(\boldsymbol{G}^{F}\right)$ est une isométrie. D'autre part, il est facile de vérifier que

$$
t_{z}^{G} \circ t_{z^{\prime}}^{G}=t_{z z^{\prime}}^{G}
$$

pour tous $z$ et $z^{\prime}$ dans $Z^{F}$. L'application $z \mapsto t_{z}^{G}$ définit donc une action de $\boldsymbol{Z}^{F}$ par isométries sur $\operatorname{Cent}\left(\boldsymbol{G}^{F}\right)$.

Soit $\widetilde{\boldsymbol{B}}_{0}$ un sous-groupe de Borel $F$-stable de $\widetilde{\boldsymbol{G}}$ et soit $\widetilde{\boldsymbol{T}}_{0}$ un tore maximal $F$-stable de $\widetilde{\boldsymbol{B}}_{0}$. On note $\boldsymbol{U}_{0}$ le radical unipotent de $\widetilde{\boldsymbol{B}}_{0}$ et on pose

$$
\boldsymbol{B}_{0}=\widetilde{\boldsymbol{B}}_{0} \cap \boldsymbol{G}, \quad \boldsymbol{T}_{0}=\widetilde{\boldsymbol{T}}_{0} \cap \boldsymbol{G} .
$$

Soit $\left(\widetilde{\boldsymbol{G}}^{*}, \widetilde{\boldsymbol{T}}_{0}^{*}, F^{*}\right)$ (resp. $\left.\left(\boldsymbol{G}^{*}, \boldsymbol{T}_{0}^{*}, F^{*}\right)\right)$ un triplet dual de $\left(\widetilde{\boldsymbol{G}}, \widetilde{\boldsymbol{T}}_{0}, F\right)$ (resp. $\left(\boldsymbol{G}, \boldsymbol{T}_{0}, F\right)$ ). L'injection canonique $i: \boldsymbol{G} \hookrightarrow \widetilde{\boldsymbol{G}}$ induit un morphisme surjectif 
de groupes $i^{*}: \widetilde{\boldsymbol{G}}^{*} \rightarrow \boldsymbol{G}^{*}$, défini sur $\mathbb{F}_{q}$ et tel que $i^{*}\left(\widetilde{\boldsymbol{T}}_{0}^{*}\right)=\boldsymbol{T}_{0}^{*}$. Le noyau de $i^{*}$ est connexe : c'est un tore central de $\widetilde{\boldsymbol{G}}^{*}$ dual de $\widetilde{\boldsymbol{G}} / \boldsymbol{G}$. En particulier, l'application $\widetilde{\boldsymbol{G}}^{* F^{*}} \rightarrow \boldsymbol{G}^{* F^{*}}$ induite par $i^{*}$ est surjective d'après le théorème de Lang. On posera

$$
A^{*}=\operatorname{Ker} i^{*} \cap \boldsymbol{D}\left(\boldsymbol{G}^{*}\right) .
$$

Soit $a \in\left(\operatorname{Ker} i^{*}\right)^{F^{*}}$. Alors $a$ induit un caractère linéaire de $\widetilde{\boldsymbol{G}}^{F} / \boldsymbol{G}^{F}$ par dualité. Ce caractère est trivial sur $\widetilde{Z}^{F}$ si et seulement si $a \in A^{* F^{*}}$ (cf. [DLM, 3.12.1]). On a donc un isomorphisme de groupes abéliens finis

$$
\boldsymbol{\omega}_{\boldsymbol{G}, F}: A^{* F^{*}} \stackrel{\sim}{\longrightarrow}\left(\widetilde{\boldsymbol{G}}^{F} / \boldsymbol{G}^{F} \cdot \widetilde{\boldsymbol{Z}}^{F}\right)^{\wedge} .
$$

Si $s$ est un élément semi-simple de $\boldsymbol{G}^{* F^{*}}$, on notera $\mathcal{E}\left(\boldsymbol{G}^{F},[s]\right)\left(\operatorname{resp} . \mathcal{E}\left(\boldsymbol{G}^{F},(s)\right)\right)$ la série de Lusztig rationnelle (resp. géométrique) associée à $s$. On notera $\phi_{s}$ le caractère linéaire de $Z^{F}$ tel que

$$
t_{z}^{G} \gamma=\phi_{s}(z) \gamma
$$

pour tous $z \in \boldsymbol{G}^{F}$ et $\gamma \in \mathcal{E}\left(\boldsymbol{G}^{F},[s]\right)$.

0.5. - Pour finir, on fixe un troisième groupe réductif connexe $\widehat{\boldsymbol{G}}$ défini sur $\mathbb{F}_{q}$. On notera encore $F: \widehat{\boldsymbol{G}} \rightarrow \widehat{\boldsymbol{G}}$ le morphisme de Frobenius. Soit $\pi: \widehat{\boldsymbol{G}} \rightarrow \boldsymbol{G}$ un morphisme de groupes algébriques défini sur $\mathbb{F}_{q}$ et tel que

1) $\operatorname{Ker} \pi$ est central dans $\widehat{\boldsymbol{G}}$;

2) $\pi(\widehat{\boldsymbol{G}})$ contient le groupe dérivé de $\boldsymbol{G}$.

On note $\widehat{\boldsymbol{Z}}$ le centre de $\widehat{\boldsymbol{G}}$. Les propriétés 1) et 2) ci-dessus montrent que $\widehat{\boldsymbol{Z}}=\pi^{-1}(\boldsymbol{Z})$ et $\boldsymbol{G}=\pi(\widehat{\boldsymbol{G}}) \cdot \boldsymbol{Z}^{0}$. On pose $\widehat{\boldsymbol{B}}_{0}=\pi^{-1}\left(\boldsymbol{B}_{0}\right)$ et $\widehat{\boldsymbol{T}}_{0}=\pi^{-1}\left(\boldsymbol{T}_{0}\right)$ et on note $\left(\widehat{\boldsymbol{G}}^{*}, \widehat{\boldsymbol{T}}_{0}^{*}, F^{*}\right)$ un triplet dual de $\left(\widehat{\boldsymbol{G}}, \widehat{\boldsymbol{T}}_{0}, F\right)$. L'application $\pi$ induit un morphisme de groupes algébriques $\pi^{*}: G^{*} \rightarrow \widehat{G}^{*}$, défini sur $\mathbb{F}_{q}$ et tel que $\pi^{*-1}\left(\widehat{\boldsymbol{T}}_{0}^{*}\right)=\boldsymbol{T}_{0}^{*}$.

\section{Quelques propriétés du groupe $\mathrm{Z} / \mathrm{Z}^{0}$}

1.1. Cuspidalité. - Soit $\boldsymbol{L}$ un sous-groupe de Levi d'un sous-groupe parabolique de $\boldsymbol{G}$. Le morphisme injectif de groupes $\boldsymbol{Z} \hookrightarrow \boldsymbol{Z}(\boldsymbol{L})$ induit un morphisme surjectif

$$
h_{\boldsymbol{L}}: \boldsymbol{Z} / \boldsymbol{Z}^{0} \longrightarrow \boldsymbol{Z}(\boldsymbol{L}) / \boldsymbol{Z}(\boldsymbol{L})^{0}
$$

( $c f$. [DLM, lemme 1.4]). Le but de ce paragraphe est d'étudier quelques propriétés du morphisme $h_{\boldsymbol{L}}$. S'il y a ambiguïté possible, on notera $h_{\boldsymbol{L}}^{G}$ le morphisme de 
groupes $h_{\boldsymbol{L}}$. Soit $\hat{\boldsymbol{L}}=\pi^{-1}(\boldsymbol{L})$. Alors $\hat{\boldsymbol{L}}$ est un sous-groupe de Levi d'un sousgroupe parabolique de $\widehat{\boldsymbol{G}}$. Le diagramme suivant

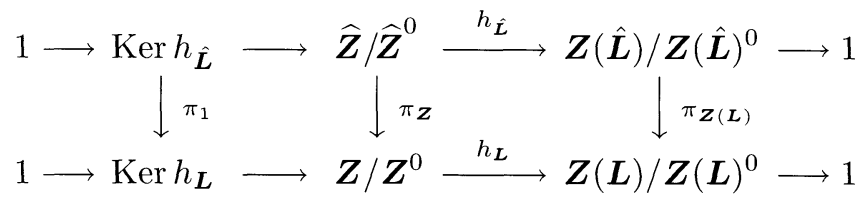

est commutatif et les lignes de ce diagramme sont des suites exactes (les applications $\pi_{1}, \pi_{\boldsymbol{Z}}$ et $\pi_{\boldsymbol{Z}(\boldsymbol{L})}$ sont induites par $\pi$ ).

Proposition 1.1.2. - Les applications $\pi_{1}, \pi_{\boldsymbol{Z}}$ et $\pi_{\boldsymbol{Z}(\boldsymbol{L})}$ sont surjectives.

Démonstration. - Montrons tout d'abord la surjectivité de $\pi_{1}$. Soit $z$ un élément de $\boldsymbol{Z}$ dont la classe dans $\boldsymbol{Z} / \boldsymbol{Z}^{0}$ appartienne au noyau de $h_{\boldsymbol{L}}$. Cela signifie que $z \in \boldsymbol{Z}(\boldsymbol{L})^{0}$. Mais $\pi\left(\boldsymbol{Z}(\hat{\boldsymbol{L}})^{0}\right) \cdot \boldsymbol{Z}^{0}=\boldsymbol{Z}(\boldsymbol{L})^{0}$; donc il existe $\hat{z} \in \boldsymbol{Z}(\hat{\boldsymbol{L}})^{0}$ et $z^{0} \in \boldsymbol{Z}^{0}$ tels que $\pi(\hat{z})=z z^{0}$. En particulier, $\hat{z} \in \widehat{\boldsymbol{Z}}$ et la classe de $\hat{z} \operatorname{dans} \widehat{\boldsymbol{Z}} / \widehat{\boldsymbol{Z}}^{0}$ appartient à Ker $h_{\hat{\boldsymbol{L}}}$. Cela montre la surjectivité de $\pi_{1}$.

La surjectivité de $\pi_{\boldsymbol{Z}}$ découle de celle de $\pi_{1}$ : il suffit de supposer que $\boldsymbol{L}$ est un tore maximal de $\boldsymbol{G}$. La surjectivité de $\pi_{\boldsymbol{Z}(\boldsymbol{L})}$ découle aussi de celle de $\pi_{\boldsymbol{Z}}$ : il suffit de supposer que $\boldsymbol{G}=\boldsymbol{L}$.

Un caractère linéaire $\zeta$ de $\boldsymbol{Z} / \boldsymbol{Z}^{0}$ est dit cuspidal si Ker $h_{\boldsymbol{L}} \nsubseteq \mathbb{K} \operatorname{Ker} \zeta$ pour tout sous-groupe de Levi $\boldsymbol{L}$ d'un sous-groupe parabolique propre de $\boldsymbol{G}$. On notera :

- $\left(\boldsymbol{Z} / \boldsymbol{Z}^{0}\right)_{\text {cus }}^{\wedge}$ l'ensemble des caractères linéaires cuspidaux de $\boldsymbol{Z} / \boldsymbol{Z}^{0}$;

- $H^{1}(F, \boldsymbol{Z})_{\text {cus }}^{\wedge}$ l'ensemble des caractères linéaires cuspidaux $F$-stables de $\boldsymbol{Z} / \boldsymbol{Z}^{0}$.

Proposition 1.1.3. - Soit $\zeta$ un caractère linéaire de $\boldsymbol{Z} / \boldsymbol{Z}^{0}$ et soit $\hat{\boldsymbol{\zeta}}=\zeta \circ \pi_{\boldsymbol{Z}}$. Alors $\zeta$ est cuspidal si et seulement si $\hat{\zeta}$ l'est.

Preuve. - Cela résulte immédiatement de la commutativité du diagramme (1.1.1) et de la proposition 1.1.2.

Corollaire 1.1.4. - Si $\left(\boldsymbol{Z} / \boldsymbol{Z}^{0}\right)_{\text {cus }}^{\wedge}$ est non vide, alors toutes les composantes irréductibles du système de racines de $\boldsymbol{G}$ sont de type $A$.

Preuve. - D'après la proposition 1.1.3, on peut supposer que $\boldsymbol{G}$ est semisimple simplement connexe. On écrit $\boldsymbol{G}=\boldsymbol{G}_{1} \times \cdots \times \boldsymbol{G}_{r}$ où $r$ est un entier naturel non nul et $\boldsymbol{G}_{1}, \ldots, \boldsymbol{G}_{r}$ sont des groupes semi-simples simplement connexes dont le système de racines est irréductible. On note $\boldsymbol{Z}_{i}$ le centre de $\boldsymbol{G}_{i}(1 \leq i \leq r)$. Alors $\boldsymbol{Z}=\boldsymbol{Z}_{1} \times \cdots \times \boldsymbol{Z}_{r}$ donc il existe des caractères linéaires $\zeta_{1}, \ldots, \zeta_{r}$ de $\boldsymbol{Z}_{1}, \ldots, \boldsymbol{Z}_{r}$ respectivement tels que $\zeta=\zeta_{1} \otimes \cdots \otimes \zeta_{r}$. 
Si $\boldsymbol{L}$ est un sous-groupe de Levi d'un sous-groupe parabolique de $\boldsymbol{G}$, alors il existe des sous-groupes de Levi $\boldsymbol{L}_{1}, \ldots, \boldsymbol{L}_{r}$ de sous-groupes paraboliques de $\boldsymbol{G}_{1}, \ldots, \boldsymbol{G}_{r}$ respectivement tels que $\boldsymbol{L}=\boldsymbol{L}_{1} \times \cdots \times \boldsymbol{L}_{r}$. Alors

$$
\operatorname{Ker} h_{\boldsymbol{L}}^{\boldsymbol{G}}=\operatorname{Ker} h_{\boldsymbol{L}_{1}}^{\boldsymbol{G}_{1}} \times \cdots \times \operatorname{Ker} h_{\boldsymbol{L}_{r}}^{\boldsymbol{G}_{r}}
$$

et donc $\zeta$ est cuspidal si et seulement si $\zeta_{1}, \ldots, \zeta_{r}$ le sont. Pour montrer le corollaire 1.1.4, on peut donc supposer que $G$ est semi-simple simplement connexe et quasi-simple. Un examen au cas par cas montre que, si $\boldsymbol{G}$ n'est pas de type $A$, alors il existe un sous-groupe de Levi $\boldsymbol{L}$ d'un sous-groupe parabolique propre de $\boldsymbol{G}$ tel que $\operatorname{Ker} h_{\boldsymbol{L}}=\{1\}$. Cela termine la démonstration.

On dira que le groupe $\boldsymbol{G}$ est de type $A$ si toutes les composantes irréductibles de son système de racines sont de type $A$.

1.2. Systèmes locaux cuspidaux sur la classe unipotente régulière. On note $C_{\text {reg }}$ ou $C_{\text {reg }}^{G}$ la classe de conjugaison des éléments unipotents réguliers de $\boldsymbol{G}$. On fixe une fois pour toutes un élément unipotent régulier $u_{1} \in C_{\mathrm{reg}}^{F}$. On suppose que $u_{1} \in \boldsymbol{U}_{0}^{F}$. Alors

$$
A_{\boldsymbol{G}}\left(u_{1}\right)=\boldsymbol{Z} / \boldsymbol{Z}^{0} \times A_{\boldsymbol{U}_{0}}\left(u_{1}\right)
$$

Remarque. - Si $p$ est bon pour $\boldsymbol{G}$, alors le groupe $C_{\boldsymbol{U}_{0}}\left(u_{1}\right)$ est connexe (cf. $[\mathrm{Sp}, 4.11])$. Dans ce cas, $A_{\boldsymbol{G}}\left(u_{1}\right) \simeq \boldsymbol{Z} / \boldsymbol{Z}^{0}$.

Le groupe $A_{\boldsymbol{G}}\left(u_{1}\right)$ est abélien d'après [Sp, 4.11] et [Lou]. Si $\zeta$ est un caractère linéaire de $\boldsymbol{Z} / \boldsymbol{Z}^{0}$, on notera $\zeta_{1}$ le caractère linéaire $\zeta \otimes 1$ de $A_{\boldsymbol{G}}\left(u_{1}\right)$. La proposition suivante est bien connue et justifie la terminologie «cuspidal» pour les caractères linéaires de $\boldsymbol{Z} / \boldsymbol{Z}^{0}$. Nous en rappelons la démonstration pour la commodité du lecteur.

Proposition 1.2.2. - Soit $\zeta$ un caractère linéaire de $\boldsymbol{Z} / \boldsymbol{Z}^{0}$. Alors $\zeta$ est cuspidal si et seulement si la paire $\left(u_{1}, \zeta_{1}\right)$ est cuspidale au sens de [L2, intro.].

Preuve. - Soit $\boldsymbol{L}$ un sous-groupe de Levi d'un sous-groupe parabolique $\boldsymbol{P}$ de $\boldsymbol{G}$ et soit $v$ un élément unipotent de $\boldsymbol{L}$. On note $\boldsymbol{U}_{\boldsymbol{P}}$ le radical unipotent de $\boldsymbol{P}$. Comme dans [L2, intro.], on pose

$$
\boldsymbol{Y}_{u_{1}, v}=\left\{g \in \boldsymbol{G} ; g^{-1} u_{1} g \in v \boldsymbol{U}_{\boldsymbol{P}}\right\} / C_{\boldsymbol{L}}^{0}(v) \boldsymbol{U}_{\boldsymbol{P}}
$$

Puisque $u_{1}$ est régulier, il va être facile de déterminer la variété $\boldsymbol{Y}_{u_{1}, v}$.

Soit $g C_{\boldsymbol{L}}^{0}(v) \boldsymbol{U}_{\boldsymbol{P}} \in \boldsymbol{Y}_{u_{1}, v}$ et soient $\boldsymbol{B}_{1}$ et $\boldsymbol{B}_{2}$ deux sous-groupes de Borel de $\boldsymbol{L}$ contenant $v$. Alors $\boldsymbol{B}_{1} \boldsymbol{U}_{\boldsymbol{P}}$ et $\boldsymbol{B}_{2} \boldsymbol{U}_{\boldsymbol{P}}$ sont deux sous-groupes de Borel de $\boldsymbol{G}$

TOME $128-2000-\mathrm{N}^{\circ} 3$ 
contenant $g^{-1} u_{1} g$, donc ils sont égaux car $u_{1}$ est régulier. Donc $\boldsymbol{B}_{1}=\boldsymbol{B}_{2}$, ce qui montre que $v$ est régulier dans $\boldsymbol{L}$. Donc si la variété $\boldsymbol{Y}_{u_{1}, v}$ est non vide, alors $v$ est régulier.

Réciproquement, supposons $v$ régulier. On déduit des résultats classiques sur les unipotents réguliers que $\boldsymbol{Y}_{u_{1}, v}$ est non vide ( $c f$. [St1, lemme 3.2 et th. 3.3]). On peut donc supposer que $u_{1} \in v \boldsymbol{U}_{\boldsymbol{P}}$. Soit $g C_{\boldsymbol{L}}^{0}(v) \boldsymbol{U}_{\boldsymbol{P}} \in \boldsymbol{Y}_{u_{1}, v}$ et soit $\boldsymbol{B}_{1}$ le sous-groupe de Borel de $\boldsymbol{L}$ contenant $v$. Alors ${ }^{g}\left(\boldsymbol{B}_{1} \boldsymbol{U}_{\boldsymbol{P}}\right)$ et $\boldsymbol{B}_{1} \boldsymbol{U}_{\boldsymbol{P}}$ sont deux sous-groupes de Borel de $\boldsymbol{G}$ contenant $u_{1}$, donc ils sont égaux, donc $g \in \boldsymbol{B}_{1} \boldsymbol{U}_{\boldsymbol{P}}$. En particulier $g \in \boldsymbol{P}$. La condition $g^{-1} u_{1} g \in v \boldsymbol{U}_{\boldsymbol{P}}$ implique alors que $g \in C_{\boldsymbol{L}}(v) \boldsymbol{U}_{\boldsymbol{P}}$. Cela montre que $\boldsymbol{Y}_{u_{1}, v}=A_{\boldsymbol{L}}(v)$. Le groupe $A_{\boldsymbol{G}}\left(u_{1}\right)$ agit sur $A_{L}(v)$ par translation à gauche. La représentation par permutation de $A_{G}\left(u_{1}\right)$ associée à $A_{\boldsymbol{L}}(v)$ contient le caractère linéaire $\zeta_{1}$ si et seulement si $\operatorname{Ker} h_{\boldsymbol{L}} \subset \operatorname{Ker} \zeta$. Cela démontre la proposition 1.2.2.

L'ensemble des classes d'isomorphismes de systèmes locaux $G$-équivariant irréductibles sur $C_{\text {reg }}$ est en bijection avec $A_{\boldsymbol{G}}\left(u_{1}\right)^{\wedge}: \operatorname{si} \zeta \in\left(\boldsymbol{Z} / \boldsymbol{Z}^{0}\right)^{\wedge}$, on notera $\mathcal{L}_{\zeta}$ le système local $\boldsymbol{G}$-équivariant sur $C_{\text {reg }}$ associé à $\zeta_{1}$. Le système local $\mathcal{L}_{\zeta}$ est $F$-stable (c'est-à-dire isomorphe à $F^{*} \mathcal{L}_{\zeta}$ ) si et seulement si le caractère linéaire $\zeta$ est $F$-stable. Pour finir, la paire $\left(C_{\text {reg }}, \mathcal{L}_{\zeta}\right)$ est cuspidale au sens de [L2, déf. 2.4] si et seulement si $\zeta$ est cuspidal d'après la proposition 1.2.2.

1.3. Centralisateurs d'éléments semi-simples. - D'après [A, 2.3], on a un isomorphisme de groupes

$$
\sigma_{\boldsymbol{G}, F}: \widetilde{\boldsymbol{G}}^{F} / \boldsymbol{G}^{F} \cdot \widetilde{\boldsymbol{Z}}^{F} \longrightarrow H^{1}(F, \boldsymbol{Z})^{\wedge} .
$$

Si $n$ est un entier naturel tel que $F^{n}$ et $F^{* n}$ agissent trivialement sur $\boldsymbol{Z} / \boldsymbol{Z}^{0}$ et $A^{*}$ respectivement, alors $\widehat{\sigma}_{\boldsymbol{G}, F^{n}} \circ \boldsymbol{\omega}_{\boldsymbol{G}, F^{n}}$ est un isomorphisme de groupes

$$
\omega: A^{*} \longrightarrow\left(\boldsymbol{Z} / \boldsymbol{Z}^{0}\right)^{\wedge}
$$

qui commute avec l'action des morphismes de Frobenius $F$ et $F^{*}$.

On fixe un élément semi-simple $s \in G^{* F^{*}}$. Alors il existe un élément semisimple $\tilde{s}$ dans $\widetilde{\boldsymbol{G}}^{* F^{*}}$ tel que $i^{*}(\tilde{s})=s$. On considère l'application

$$
\begin{aligned}
C_{G^{*}}(s) & \longrightarrow A^{*}, \\
g & \longmapsto \tilde{g} \tilde{s} \tilde{g}^{-1} \tilde{s}^{-1}=[\tilde{g}, \tilde{s}]
\end{aligned}
$$

où, pour tout $g \in C_{\boldsymbol{G}^{*}}(s), \tilde{g}$ désigne un élément de $\widetilde{\boldsymbol{G}}^{*}$ tel que $i^{*}(\tilde{g})=g$. Puisque $A^{*}$ est central, cette application ne dépend pas du choix de $\tilde{s}$ et $\tilde{g}$ et c'est un morphisme de groupes. Le noyau de ce morphisme est $i^{*}\left(C_{\widetilde{G}^{*}}(\tilde{s})\right)$. 
Puisque $C_{\widetilde{\boldsymbol{G}}^{*}}(\tilde{s})$ est connexe ( $c f$. par exemple [DL, cor. 5.24]), on a, d'après [DM3, prop. 2.3], $i^{*}\left(C_{\widetilde{\boldsymbol{G}}^{*}}(\tilde{s})\right)=C_{\boldsymbol{G}^{*}}^{0}(s)$. Par conséquent, ce morphisme induit un morphisme injectif

$$
\widetilde{\omega}_{s}: A_{G^{*}}(s) \longrightarrow A^{*}
$$

qui, par composition avec l'isomorphisme $\omega$ induit un morphisme injectif

$$
\omega_{s}: A_{\boldsymbol{G}^{*}}(s) \longrightarrow\left(\boldsymbol{Z} / \boldsymbol{Z}^{0}\right)^{\wedge}
$$

qui commute aux actions de $F$ et $F^{*}$. En particulier, $A_{G^{*}}(s)$ est abélien et $\omega_{s}$ induit cinq autres morphismes de groupes

$$
\left\{\begin{array}{l}
\omega_{s}^{0}: A_{\boldsymbol{G}^{*}}(s)^{F^{*}} \longrightarrow H^{1}(F, \boldsymbol{Z})^{\wedge} \\
\omega_{s}^{1}: H^{1}\left(F^{*}, A_{\boldsymbol{G}^{*}}(s)\right) \longrightarrow\left(\boldsymbol{Z}^{F} / \boldsymbol{Z}^{\circ F}\right)^{\wedge}, \\
\widehat{\omega}_{s}: \boldsymbol{Z} / \boldsymbol{Z}^{0} \longrightarrow A_{\boldsymbol{G}^{*}}(s)^{\wedge}, \\
\widehat{\omega}_{s}^{0}: H^{1}(F, \boldsymbol{Z}) \longrightarrow\left(A_{\boldsymbol{G}^{*}}(s)^{F^{*}}\right)^{\wedge}, \\
\widehat{\omega}_{s}^{1}: \boldsymbol{Z}^{F} / \boldsymbol{Z}^{\circ F} \longrightarrow H^{1}\left(F^{*}, A_{\boldsymbol{G}^{*}}(s)\right)^{\wedge} .
\end{array}\right.
$$

Le morphisme $\omega_{s}^{0}$ est injectif et les morphismes $\widehat{\omega}_{s}$ et $\widehat{\omega}_{s}^{0}$ sont surjectifs. S'il y a risque d'ambiguité, on notera $\omega_{\boldsymbol{G}, s}$ le morphisme $\omega_{s}$ et similairement pour les autres.

On rappelle le lemme suivant ( $c f$. par exemple [A, lemme 1.2.1]) :

LEMme 1.3.3. - Via $\widetilde{\omega}_{s}$, on a un isomorphisme

$$
\begin{aligned}
A_{\boldsymbol{G}^{*}}(s) & \simeq\left\{z \in A^{*} \mid \tilde{s} \text { et } \tilde{s} z \text { sont conjugués dans } \widetilde{\boldsymbol{G}}^{*}\right\} \\
& \simeq\left\{z \in \operatorname{Ker} i^{*} \mid \tilde{s} \text { et } \tilde{s} z \text { sont conjugués dans } \widetilde{\boldsymbol{G}}^{*}\right\}
\end{aligned}
$$

Si $\alpha \in H^{1}\left(F^{*}, A_{G^{*}}(s)\right)$, on notera $g_{\alpha}$ un élément de $\boldsymbol{G}^{*}$ tel que $g_{\alpha}^{-1} F\left(g_{\alpha}\right)$ appartienne à $C_{\boldsymbol{G}^{*}}(s)$ et représente $\alpha$. Alors $s_{\alpha}=g_{\alpha} s g_{\alpha}^{-1}$ est un élément semisimple de $\boldsymbol{G}^{* F^{*}}$ géométriquement conjugué à $s$. D'après le théorème de Lang, les $s_{\alpha}\left(\alpha \in H^{1}\left(F^{*}, A_{G^{*}}(s)\right)\right.$ forment un système de représentants de l'ensemble des classes de conjugaison rationnelles contenues dans $(s)^{F^{*}}$. Par conséquent, on a

$$
\mathcal{E}\left(\boldsymbol{G}^{F},(s)\right)=\coprod_{\alpha \in H^{1}} \coprod_{\left(F^{*}, A_{\mathbf{G}^{*}}(s)\right)} \mathcal{E}\left(\boldsymbol{G}^{F},\left[s_{\alpha}\right]\right) .
$$

D'autre part, le groupe $C_{\boldsymbol{G}^{*}}\left(s_{\alpha}\right)$ est isomorphe à $C_{\boldsymbol{G}^{*}}(s)$ muni de l'endomorphisme de Frobenius $\dot{\alpha} F^{*}$, où $\dot{\alpha}$ désigne la classe de $g_{\alpha}^{-1} F\left(g_{\alpha}\right)$ dans $A_{G^{*}}(s)$. Puisque $A_{\boldsymbol{G}^{*}}(s)$ est abélien, on peut identifier $A_{\boldsymbol{G}^{*}}(s)$ et $A_{\boldsymbol{G}^{*}}\left(s_{\alpha}\right)$ car les morphismes de Frobenius $F^{*}$ et $\dot{\alpha} F^{*}$ coïncident sur $A_{\boldsymbol{G}^{*}}(s)$. Le lemme suivant est démontré lorsque $F$ agit trivialement sur $\boldsymbol{Z} / \boldsymbol{Z}^{0}$ dans [A, lemme 2.4.1, (ii)]. Le cas général se démontre exactement de la même manière.

TOME $128-2000-\mathrm{N}^{\circ} 3$ 
Lemme 1.3.4. - Soit $\alpha \in H^{1}\left(F^{*}, A_{G^{*}}(s)\right)$. Alors $\phi_{s_{\alpha}}=\phi_{s} \widehat{\omega}_{s}^{1}(\alpha)$.

1.4. Cuspidalité et éléments semi-simples. - On reprend les notations du paragraphe précédent.

\section{DÉFINITION 1.4.1.}

- L'élément semi-simple $s$ est dit géométriquement cuspidal (dans $\boldsymbol{G}^{*}$ ) si il existe un élément $a \in A_{G^{*}}(s)$ tel que $\omega_{s}(a)$ soit un caractère linéaire cuspidal de $\boldsymbol{Z} / \boldsymbol{Z}^{0}$.

- L'élément semi-simple $s$ est dit rationnellement cuspidal (dans $\boldsymbol{G}^{*}$ ) s'il existe un élément $a \in A_{G^{*}}(s)^{F^{*}}$ tel que $\omega_{s}^{0}(a)$ soit un caractère linéaire cuspidal de $H^{1}(F, \boldsymbol{Z})$.

Si $s$ est rationnellement cuspidal, alors il est géométriquement cuspidal. De plus, il résulte du corollaire 1.1.4 que :

Proposition 1.4.2. - Si $G^{*}$ contient un élément géométriquement cuspidal, alors $\boldsymbol{G}$ est de type $A$.

Soit $\boldsymbol{L}$ un sous-groupe de Levi d'un sous-groupe parabolique de $\boldsymbol{G}$ et soit $\boldsymbol{L}^{*}$ un sous-groupe de Levi d'un sous-groupe parabolique de $\boldsymbol{G}^{*}$ dual de $\boldsymbol{L}$. Si $\boldsymbol{L}$ est $F$-stable, on choisit $\boldsymbol{L}^{*}$ rationnel et de sorte que $\left(\boldsymbol{L}^{*}, F^{*}\right)$ soit dual de $(\boldsymbol{L}, F)$. Supposons que $s \in \boldsymbol{L}^{*}$. Le morphisme $A_{\boldsymbol{L}^{*}}(s) \longrightarrow A_{\boldsymbol{G}^{*}}(s)$ est injectif et le diagramme suivant

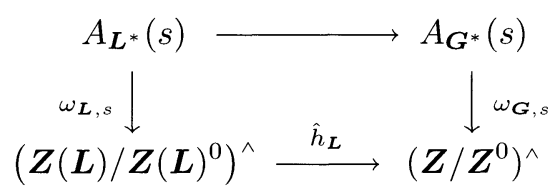

est commutatif. De plus tous les morphismes de ce diagramme sont injectifs et, si $\boldsymbol{L}$ est $F$-stable, ils commutent aux actions de $F$ et $F^{*}$. La proposition suivante résulte immédiatement de la commutativité de ce diagramme.

Proposition 1.4.4. - Supposons s géométriquement cuspidal. Alors pour tout sous-groupe de Levi $\boldsymbol{L}^{*}$ d'un sous-groupe parabolique de $\boldsymbol{G}^{*}$ tel que $s \in \boldsymbol{L}^{*}$, on a $\left|A_{L^{*}}(s)\right|<\left|A_{\boldsymbol{G}^{*}}(s)\right|$ ou, de manière équivalente, le morphisme $A_{\boldsymbol{L}^{*}}(s) \rightarrow A_{\boldsymbol{G}^{*}}(s)$ n'est pas surjectif.

Si s est rationnellement cuspidal, alors, pour tout sous-groupe de Levi rationnel $\boldsymbol{L}^{*}$ d'un sous-groupe parabolique de $\boldsymbol{G}^{*}$ tel que $s \in \boldsymbol{L}^{*}$, on a $\left|A_{\boldsymbol{L}^{*}}(s)^{F^{*}}\right|<$ $\left|A_{G^{*}}(s)^{F^{*}}\right|$ ou, de manière équivalente, le morphisme $A_{L^{*}}(s)^{F^{*}} \rightarrow A_{\boldsymbol{G}^{*}}(s)^{F^{*}}$ n'est pas surjectif.

Corollaire 1.4.5. - Un élément semi-simple géométriquement ou rationnellement cuspidal est régulier. 
Preuve. - Voir [B2, lemme 3.2.9].

Remarque. - La définition 1.4.1 diffère de la définition d'élément semisimple cuspidal donnée dans [B2, déf. 3.2.8]. La proposition 1.4.4 montre qu'un élément géométriquement cuspidal (resp. rationnellement cuspidal) au sens de la définition 1.4.1 est cuspidal au sens de [B2, déf. 3.2.8].

On pose $\hat{s}=\pi^{*}(s)$. D'après [DM3, prop. 2.3], on a

$$
\pi^{*}\left(C_{\boldsymbol{G}^{*}}^{0}(s)\right)=C_{\widehat{\boldsymbol{G}}^{*}}^{0}(\hat{s}) \cdot \boldsymbol{Z}\left(\widehat{\boldsymbol{G}}^{*}\right)^{0} .
$$

Cela implique que le morphisme

$$
\pi_{s}^{*}: A_{\boldsymbol{G}^{*}}(s) \longrightarrow A_{\widehat{\boldsymbol{G}}^{*}}(\hat{s})
$$

induit par $\pi^{*}$ est injectif. De plus, le diagramme suivant est commutatif :

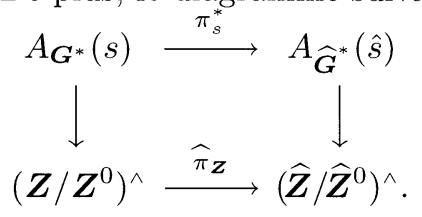

Dans ce diagramme, toutes les applications sont injectives et commutent à l'action de $F$ et $F^{*}$. Mieux, si on identifie tous les groupes de ce diagramme à des sous-groupes de $\left(\widehat{\boldsymbol{Z}} / \widehat{\boldsymbol{Z}}^{0}\right)^{\wedge}$, on a

$$
A_{\boldsymbol{G}^{*}}(s)=A_{\widehat{\boldsymbol{G}}^{*}}(\hat{s}) \cap\left(\boldsymbol{Z} / \boldsymbol{Z}^{0}\right)^{\wedge}
$$

( $c f$. lemme 1.3.3). La proposition 1.1.3 et la commutativité du diagramme (1.4.6) impliquent la proposition suivante :

Proposition 1.4.8. - Si s est géométriquement (resp. rationnellement) cuspidal dans $\boldsymbol{G}^{*}$ alors $\hat{s}$ est géométriquement (resp. rationnellement) cuspidal dans $\widehat{\boldsymbol{G}}^{*}$.

Proposition 1.4.9. - Soit s un élément géométriquement cuspidal de $\boldsymbol{G}^{*}$. Alors $\omega_{s}: A_{G^{*}}(s) \rightarrow\left(\boldsymbol{Z} / \boldsymbol{Z}^{0}\right)^{\wedge}$ est un isomorphisme.

Preuve. - D'après 1.4.7 et la proposition 1.4.8, on peut supposer que $\boldsymbol{G}$ est semi-simple simplement connexe. Par produit, on peut aussi supposer que $G$ est quasi-simple. Puisque $\boldsymbol{G}$ est nécessairement de type $A$ ( $c f$. prop. 1.4.2), on peut supposer que $\boldsymbol{G}=\mathrm{SL}_{n}(\mathbb{F})$ pour un entier naturel non nul $n$. En particulier, $G^{*}=\mathrm{PGL}_{n}(\mathbb{F})$.

Dans la preuve de [B2, lemme 3.2.10], il est prouvé qu'alors, nécessairement, $p$ ne divise pas $n$ et $s$ est conjugué à l'image dans $G^{*}$ de la matrice $\operatorname{diag}\left(1, \zeta, \ldots, \zeta^{n-1}\right)$ où $\zeta$ est une racine primitive $n$-ième de l'unité. Le groupe $A_{G^{*}}(s)$ est alors cyclique d'ordre $n$, ce qui montre le résultat.

$$
\text { TOME } 128-2000-\mathrm{N}^{\circ} 3
$$


1.5. Action de $\boldsymbol{H}^{\mathbf{1}}(\boldsymbol{F}, \boldsymbol{Z})$ sur $\operatorname{Irr} \boldsymbol{G}^{\boldsymbol{F}}$. - On rappelle que l'on a un isomorphisme de groupes

$$
\sigma_{\boldsymbol{G}}: \widetilde{\boldsymbol{G}}^{F} / \boldsymbol{G}^{F} \cdot \widetilde{\boldsymbol{Z}}^{F} \longrightarrow H^{1}(F, \boldsymbol{Z})
$$

défini de la manière suivante $(c f$. $[\mathrm{A}, 2.3])$. Si $\tilde{g} \in \widetilde{\boldsymbol{G}}^{F}$, alors il existe $g \in \boldsymbol{G}$ et $\tilde{z} \in \widetilde{\boldsymbol{Z}}$ tels que $\tilde{g}=g \tilde{z}$. Puisque $F(\tilde{g})=\tilde{g}$, on a $g^{-1} F(g)=\tilde{z} F(\tilde{z})^{-1} \in \boldsymbol{G} \cap \widetilde{\boldsymbol{Z}}=\boldsymbol{Z}$. L'image par $\sigma_{\boldsymbol{G}}$ de la classe de $\tilde{g}$ dans $\widetilde{\boldsymbol{G}}^{F} / \boldsymbol{G}^{F} \cdot \widetilde{\boldsymbol{Z}}^{F}$ est alors égale à la classe de $g^{-1} F(g)$ dans $H^{1}(F, \boldsymbol{Z})$. Le groupe $\widetilde{\boldsymbol{G}}^{F} / \boldsymbol{G}^{F} \cdot \widetilde{\boldsymbol{Z}}^{F}$ agit sur $\operatorname{Irr} \boldsymbol{G}^{F}$ par conjugaison donc, via $\sigma_{G}$, le groupe $H^{1}(F, \boldsymbol{Z})$ agit sur $\operatorname{Irr} \boldsymbol{G}^{F}$. On peut étendre cette action par linéarité au $\overline{\mathbb{Q}}_{\ell}$-espace vectoriel $\operatorname{Cent}\left(\boldsymbol{G}^{F}\right)$. Si $c \in H^{1}(F, \boldsymbol{Z})$, on notera $\tilde{g}_{c}$ un élément de $\widetilde{\boldsymbol{G}}^{F}$ dont l'image par le morphisme composé $\widetilde{\boldsymbol{G}}^{F} \rightarrow \widetilde{\boldsymbol{G}}^{F} / \boldsymbol{G}^{F} \cdot \widetilde{\boldsymbol{Z}}^{F} \rightarrow$ $H^{1}(F, \boldsymbol{Z})$ est égale à $c$. On pose alors

$$
\tau_{c}^{\boldsymbol{G}} \gamma=\gamma \circ\left(\operatorname{int} \tilde{g}_{c}\right)^{-1}
$$

pour toute fonction centrale $\gamma$ sur $\boldsymbol{G}^{F}$. L'application $\tau_{c}^{G}: \operatorname{Cent}\left(\boldsymbol{G}^{F}\right) \rightarrow$ $\operatorname{Cent}\left(\boldsymbol{G}^{F}\right)$ est une isométrie qui permute l'ensemble des caractères irréductibles de $\boldsymbol{G}^{F}$. De plus

pour tous $c$ et $d$ dans $H^{1}(F, Z)$.

$$
\tau_{c}^{G} \circ \tau_{d}^{G}=\tau_{c d}^{G}
$$

1.6. Sous-groupes de Levi. - Soit $\boldsymbol{L}$ un sous-groupe de Levi rationnel d'un sous-groupe parabolique $\boldsymbol{P}$ de $\boldsymbol{G}$. Le morphisme surjectif $h_{\boldsymbol{L}}$ induit un morphisme surjectif de groupes $\boldsymbol{h}_{\boldsymbol{L}}: H^{1}(F, \boldsymbol{Z}) \rightarrow H^{1}(F, \boldsymbol{Z}(\boldsymbol{L}))$. S'il y a ambiguïté, on notera $\boldsymbol{h}_{\boldsymbol{L}}^{G}$ le morphisme $\boldsymbol{h}_{\boldsymbol{L}}$.

On a un isomorphisme

$$
\sigma_{\boldsymbol{L}}: \widetilde{\boldsymbol{L}}^{F} / \boldsymbol{L}^{F} \cdot \boldsymbol{Z}(\widetilde{\boldsymbol{L}})^{F} \longrightarrow H^{1}(F, \boldsymbol{Z}(\boldsymbol{L})) .
$$

D'autre part, le morphisme de groupes canonique $\widetilde{\boldsymbol{L}}^{F} \hookrightarrow \widetilde{\boldsymbol{G}}^{F}$ induit un isomorphisme

$$
\sigma_{\boldsymbol{L}}^{\boldsymbol{G}}: \widetilde{\boldsymbol{L}}^{F} / \boldsymbol{L}^{F} \cdot \widetilde{\boldsymbol{Z}}^{F} \longrightarrow H^{1}(F, \boldsymbol{Z}) .
$$

Cela signifie que, lorsque cela sera nécessaire, on pourra supposer que $\tilde{g}_{c} \in \boldsymbol{L}^{F}$ pour tout $c \in H^{1}(F, \boldsymbol{Z})$. Par suite, le diagramme

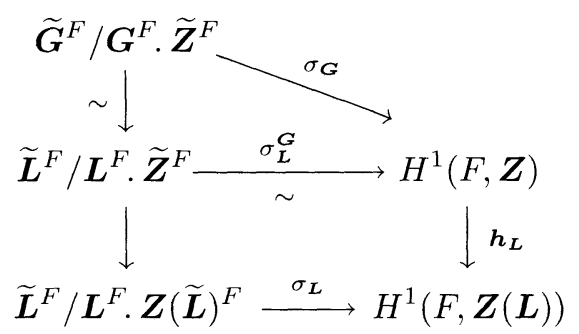

est commutatif. 


\section{Opérateur de torsion}

2.1. Définition. - Soit $g \in \boldsymbol{G}^{F}$. Par le théorème de Lang, il existe $x \in \boldsymbol{G}$ tel que $x^{-1} F(x)=g$. Alors $g^{\prime}=F(x) x^{-1} \in G^{F}$ et il est facile de vérifier que l'application

$$
\begin{aligned}
N_{F / F}^{G}: \operatorname{Conj}\left(\boldsymbol{G}^{F}\right) & \longrightarrow \operatorname{Conj}\left(\boldsymbol{G}^{F}\right), \\
{[g] } & \longmapsto\left[g^{\prime}\right]
\end{aligned}
$$

est bien définie et bijective. En particulier, elle induit une isométrie

$$
\begin{aligned}
\operatorname{Sh}_{F / F}^{G}: \operatorname{Cent}\left(\boldsymbol{G}^{F}\right) & \longrightarrow \operatorname{Cent}\left(\boldsymbol{G}^{F}\right), \\
\eta & \longmapsto \eta \circ N_{F / F}^{\boldsymbol{G}}
\end{aligned}
$$

appelée opérateur de torsion : la convention choisie ici diffère légèrement de celles de [D], [Sh1], [Sh2] ou [Sh3]. D'autre part, cet opérateur est souvent noté $t_{1}^{*}$ (cf. [A], [Sh1] ou [Sh2]). Par contre, cette convention est la même que celle de Digne et Michel dans [DM1]. Le lecteur pourra d'ailleurs se référer à [DM1] pour les propriétés classiques de l'opérateur de torsion $\mathrm{Sh}_{F / F}^{G}$.

Si $g \in G^{F}$ et si $g^{\prime} \in N_{F / F}^{G}([g])$, on fera souvent l'abus de notation suivant : $g^{\prime}=N_{F / F}^{G}(g)$.

Si $c \in H^{1}(F, \boldsymbol{Z})$, alors

$$
\tau_{c}^{\boldsymbol{G}} \circ \mathrm{Sh}_{F / F}^{\boldsymbol{G}}=\mathrm{Sh}_{F / F}^{\boldsymbol{G}} \circ \tau_{c}^{\boldsymbol{G}} .
$$

Cela résulte du fait que $\tau_{c}^{G}$ est induit par un automorphisme de $\boldsymbol{G}$ défini sur $\mathbb{F}_{q}$.

2.2. Opérateur de torsion et action de $\boldsymbol{H}^{\mathbf{1}}(\boldsymbol{F}, \boldsymbol{Z})$. - Si $z \in \boldsymbol{Z}$, on notera $\bar{z}$ la classe de $z$ dans $H^{1}(F, \boldsymbol{Z})$. Il est possible, dans certains cas particuliers, de calculer l'image d'un élément de $G^{F}$ par $N_{F / F}^{G}$ :

Lemme 2.2.1. - Soit $g$ un élément de $\boldsymbol{G}^{F}$ tel que $g \in C_{\boldsymbol{G}}^{0}(g)$ et soit $z \in \boldsymbol{Z}^{F}$. Alors

$$
N_{F / F}^{G}(z g)=\tilde{g}_{\bar{z}}(z g) \tilde{g}_{\bar{z}}^{-1}
$$

Preuve. - D'après le théorème de Lang, il existe $x \in \boldsymbol{G}$ et $\tilde{z} \in \widetilde{\boldsymbol{Z}}$ tels que $z=x^{-1} F(x)=\tilde{z}^{-1} F(\tilde{z})$. On pose $\tilde{g}=x \tilde{z}^{-1}$. Alors $\tilde{g} \in \widetilde{\boldsymbol{G}}^{F}$ et on peut supposer que $\tilde{g}=\tilde{g}_{\bar{z}}$. Puisque $g \in C_{\boldsymbol{G}}^{0}(g)$, il existe, toujours d'après le théorème de Lang, un élément $y \in C_{\boldsymbol{G}}^{0}(g)$ tel que $g=y^{-1} F(y)$. On a alors

$$
z g=z y^{-1} F(y)=y^{-1} z F(y)=(x y)^{-1} F(x y)
$$

donc

$$
\begin{aligned}
N_{F / F}^{G}(z g) & =F(x y) y^{-1} x^{-1}=F(x) y\left(y^{-1} F(y)\right) y^{-1} x^{-1} \\
& =x x^{-1} F(x) g x^{-1}=\tilde{g}(z g) \tilde{g}^{-1},
\end{aligned}
$$

la troisième égalité résultant du fait que $y$ et $g=y^{-1} F(y)$ commutent.

$$
\text { TOME } 128-2000-\mathrm{N}^{\circ} 3
$$


Remarque. - Comme cas particulier (et trivial) du lemme 2.2.1 précédent, on a que $N_{F / F}^{G}(g)=g$ pour tout élément $g \in G^{F}$ tel que $g \in C_{G}^{0}(g)$.

Corollaire 2.2.2. - Supposons que le nombre premier p est bon pour $\boldsymbol{G}$. Soit $u$ un élément unipotent de $\boldsymbol{G}^{F}$ et soit $z \in \boldsymbol{Z}^{F}$. Alors

$$
N_{F / F}^{\boldsymbol{G}}(z u)=\tilde{g}_{\bar{z}}(z u) \tilde{g}_{\bar{z}}^{-1}
$$

Preuve. - En effet, si $p$ est bon pour $\boldsymbol{G}$ et si $u$ est un élément unipotent de $\boldsymbol{G}$, alors $u \in C_{\boldsymbol{G}}^{0}(u)(c f .[$ SpSt, 3.15]) et il suffit d'appliquer le lemme 2.2.1.

Soit $\widetilde{\boldsymbol{P}}$ un sous-groupe parabolique $F$-stable de $\widetilde{\boldsymbol{G}}$ et soit $\widetilde{\boldsymbol{L}}$ un sous-groupe de Levi $F$-stable de $\widetilde{\boldsymbol{P}}$. On pose

$$
\boldsymbol{P}=\widetilde{\boldsymbol{P}} \cap \boldsymbol{G}, \quad \boldsymbol{L}=\widetilde{\boldsymbol{L}} \cap \boldsymbol{G} .
$$

Alors $\boldsymbol{P}$ est un sous-groupe parabolique $F$-stable de $\boldsymbol{G}$ et $\boldsymbol{L}$ est un sous-groupe de Levi $F$-stable de $\boldsymbol{P}$. On note $\boldsymbol{U}$ le radical unipotent de $\boldsymbol{P}$ et on note $\lambda$ un caractère de $\boldsymbol{U}^{F}$. On pose alors

$$
\Gamma_{\lambda}^{G}=\operatorname{Ind}_{U^{F}}^{G^{F}} \lambda
$$

Pour tout caractère linéaire $\phi: Z^{F} \rightarrow \overline{\mathbb{Q}}_{\ell}{ }^{\times}$, on pose

$$
\Gamma_{\phi, \lambda}^{G}=\operatorname{Ind} \boldsymbol{Z}^{F} \times \boldsymbol{U}^{F}(\phi \otimes \lambda)
$$

Si $(z, \zeta) \in \mathcal{M}^{\vee}\left(\boldsymbol{Z} / \boldsymbol{Z}^{0}, F\right)$, on pose

$$
\widehat{\boldsymbol{\Gamma}}_{\phi, \lambda,(z, \zeta)}^{\boldsymbol{G}}=\sum_{(c, \xi) \in \mathcal{M}\left(\boldsymbol{Z} / \boldsymbol{Z}^{0}, F\right)}\{(z, \zeta),(c, \xi)\} \tau_{c}^{\boldsymbol{G}} \boldsymbol{\Gamma}_{\phi \xi, \lambda}^{\boldsymbol{G}}
$$

Il est facile de vérifier que, pour tout $(c, \xi) \in \mathcal{M}\left(\boldsymbol{Z} / \boldsymbol{Z}^{0}, F\right)$, on a

$$
\tau_{c}^{G} \boldsymbol{\Gamma}_{\phi \xi, \lambda}^{\boldsymbol{G}}=\sum_{(z, \zeta) \in \mathcal{M}^{\vee}\left(\boldsymbol{Z} / \boldsymbol{Z}^{0}, F\right)} \overline{\{(z, \zeta),(c, \xi)\}} \widehat{\boldsymbol{\Gamma}}_{\phi, \lambda,(z, \zeta)}^{\boldsymbol{G}}
$$

Proposition 2.2.5. - Supposons que $p$ est bon pour $\boldsymbol{G}$. Soit $(z, \zeta) \in$ $\mathcal{M}^{\vee}\left(\boldsymbol{Z} / \boldsymbol{Z}^{0}, F\right)$. Alors

$$
\operatorname{Sh}_{F / F}^{\boldsymbol{G}} \widehat{\boldsymbol{\Gamma}}_{\phi, \lambda,(z, \zeta)}^{\boldsymbol{G}}=\zeta(\bar{z})^{-1} \widehat{\boldsymbol{\Gamma}}_{\phi, \lambda,(z, \zeta)}^{\boldsymbol{G}}
$$


Preuve. - On notera $\mathbf{1}_{z}$ la fonction centrale sur $\boldsymbol{Z}^{F}$ qui vaut 1 sur les éléments de $\boldsymbol{Z}^{F}$ représentant $z \in\left(\boldsymbol{Z} / \boldsymbol{Z}^{0}\right)^{F}$ et 0 ailleurs. On pose aussi :

$$
\Gamma=\sum_{\xi \in\left(\boldsymbol{Z}^{\left.F / \boldsymbol{Z}^{\circ F}\right)^{\wedge}}\right.} \xi(z) \boldsymbol{\Gamma}_{\phi \xi, \lambda}^{\boldsymbol{G}} .
$$

Il est alors facile de vérifier que

$$
\Gamma=\frac{\left|\boldsymbol{Z}^{F}\right|}{\left|\boldsymbol{Z}^{\circ F}\right|} \operatorname{Ind}_{\boldsymbol{Z}^{F} \times \boldsymbol{U}^{F}}^{\boldsymbol{G}^{F}}\left(\phi \cdot \mathbf{1}_{z^{-1}} \otimes \lambda\right) .
$$

Soit $g$ un élément de $\boldsymbol{G}^{F}$ tel que $\Gamma(g) \neq 0$. Alors $g=z^{\prime} u$ où $z^{\prime} \in Z^{F}$ représente $z^{-1}$ et $u$ est un élément unipotent de $\boldsymbol{G}^{F}$. Il résulte alors du corollaire 2.2 .2 que $N_{F / F}^{G}(g)=\tilde{g}_{\bar{z}}^{-1} g \tilde{g}_{\bar{z}}$ où $\bar{z}$ désigne la classe de $z$ dans $H^{1}(F, \boldsymbol{Z})$. Cela implique que

$$
\operatorname{Sh}_{F / F}^{G} \Gamma=\tau_{\bar{z}}^{G} \Gamma
$$

Le résultat découle alors du fait que

$$
\widehat{\boldsymbol{\Gamma}}_{\phi, \lambda,(z, \zeta)}^{\boldsymbol{G}}=\frac{1}{\left|H^{1}(F, \boldsymbol{Z})\right|} \sum_{c \in H^{1}(F, \boldsymbol{Z})} \zeta(c) \tau_{c}^{\boldsymbol{G}} \Gamma
$$

et de l'égalité 2.1.3.

\section{Caractères de Gel'fand-Graev}

On notera $D_{G}: \mathbb{Z} \operatorname{Irr} G^{F} \rightarrow \mathbb{Z} \operatorname{Irr} G^{F}$ l'opérateur de dualité d'Alvis-Curtis ( $c f$. par exemple [DM3, déf. 8.8] pour la définition de $D_{G}$ ).

3.1. Définition. - On fixe un caractère linéaire régulier $\psi: \boldsymbol{U}_{0}^{F} \rightarrow \overline{\mathbb{Q}}_{\ell}{ }^{\times}$ ( $c f$. [DLM, déf. 2.3] pour la définition d'un caractère régulier de $\boldsymbol{U}_{0}^{F}$ ). On pose

$$
\Gamma_{\psi}^{\boldsymbol{G}}=\operatorname{Ind}_{\boldsymbol{U}_{0}^{F}}^{\boldsymbol{G}^{F}} \psi
$$

Si $\phi$ est un caractère linéaire de $\boldsymbol{Z}^{F}$, on pose

$$
\Gamma_{\phi, \psi}^{\boldsymbol{G}}=\operatorname{Ind}_{\boldsymbol{Z}^{F} \times \boldsymbol{U}_{0}^{F}}^{\boldsymbol{G}^{F}}(\phi \otimes \psi) .
$$

Alors

$$
\Gamma_{\psi}^{\boldsymbol{G}}=\sum_{\phi \in\left(\boldsymbol{Z}^{F}\right)^{\wedge}} \Gamma_{\phi, \psi}^{\boldsymbol{G}}
$$

TOME $128-2000-\mathrm{N}^{\circ} 3$ 
Si $c \in H^{1}(F, \boldsymbol{Z})$, on choisit $\tilde{g}_{c}$ dans $\widetilde{\boldsymbol{T}}_{0}^{F}$ et on pose

$$
\psi_{c}=\psi \circ\left(\operatorname{int} \tilde{g}_{c}\right)^{-1}
$$

D'après [DLM, th. 2.4], les $\psi_{c}\left(c \in H^{1}(F, \boldsymbol{Z})\right)$ forment un systèmes de représentants de l'ensemble des $\boldsymbol{T}_{0}^{F}$-orbites dans l'ensemble des caractères réguliers de $\boldsymbol{U}_{0}^{F}$. On a de plus

$$
\Gamma_{\psi_{c}}^{\boldsymbol{G}}=\tau_{c}^{\boldsymbol{G}} \Gamma_{\psi}^{\boldsymbol{G}}
$$

pour tout $c \in H^{1}(F, \boldsymbol{Z})$. Les caractères $\Gamma_{\psi_{c}}^{G}$ sont appelés les caractères de Gel'fand-Graev de $\boldsymbol{G}$.

La proposition suivante est à la fois une conséquence et une très légère généralisation de [DLM, th 2.12].

Proposition 3.1.3. - Soient c et d deux éléments de $H^{1}(F, \boldsymbol{Z})$ et $\phi$ un caractère linéaire de $\boldsymbol{Z}^{F}$. Alors

$$
\left\langle D_{G^{G}} \Gamma_{\phi, \psi_{c}}^{\boldsymbol{G}}, \Gamma_{\phi, \psi_{d}}^{\boldsymbol{G}}\right\rangle_{\boldsymbol{G}^{F}}= \begin{cases}\eta_{\boldsymbol{G}} & \text { si } c=d \\ 0 & \text { sinon }\end{cases}
$$

Preuve. - Soit $\psi_{c}^{*}$ la fonction centrale sur $\boldsymbol{U}_{0}^{F}$ définie dans [DLM, preuve du th. 2.12 , p. 165, ligne 3] (elle est notée $\phi$ dans [DLM] et on ne peut ici réutiliser cette notation!). Elle vérifie en particulier

$$
D_{G} \Gamma_{\psi_{c}}^{G}=\operatorname{Ind}_{U_{0}^{F}}^{G^{F}} \psi_{c}^{*}
$$

En décomposant suivant l'action de $\boldsymbol{Z}^{F}$ via les $t_{z}^{G}\left(z \in \boldsymbol{Z}^{F}\right)$, on obtient

$$
D_{\boldsymbol{G}} \Gamma_{\phi, \psi_{c}}^{\boldsymbol{G}}=\operatorname{Ind}_{\boldsymbol{Z}^{F} \times \boldsymbol{U}_{0}^{F}}^{\boldsymbol{G}^{F}}\left(\phi \otimes \psi_{c}^{*}\right)
$$

La formule de Mackey pour l'induction et la restriction classique implique que

$$
\begin{aligned}
\left\langle D_{\boldsymbol{G}} \Gamma_{\phi, \psi_{c}}^{\boldsymbol{G}}, \Gamma_{\phi, \psi_{d}}^{\boldsymbol{G}}\right\rangle_{\boldsymbol{G}^{F}} & =\sum_{n \in N^{F} / \boldsymbol{Z}^{F}}\left\langle\phi \otimes \psi_{c}^{*}, \phi \otimes{ }^{n} \psi_{d}\right\rangle_{\boldsymbol{Z}^{F} \times\left(\boldsymbol{U}_{0}^{F} \cap^{n} \boldsymbol{U}_{0}^{F}\right)} \\
& =\sum_{n \in N^{F} / \boldsymbol{Z}^{F}}\left\langle\psi_{c}^{*},{ }^{n} \psi_{d}\right\rangle_{\boldsymbol{U}_{0}^{F} \cap^{n} \boldsymbol{U}_{0}^{F}}
\end{aligned}
$$

où $\boldsymbol{N}$ est le normalisateur de $\boldsymbol{T}_{0}$ dans $\boldsymbol{G}$. La conclusion s'obtient alors en suivant mot à mot le même argument que dans [DLM, fin de la preuve du th. 2.12, p. 165]. 
3.2. Caractères réguliers. - Soit $s$ un élément semi-simple de $\boldsymbol{G}^{* F^{*}}$. On fixe un sous-groupe de Borel $F^{*}$-stable $\boldsymbol{B}_{1}^{*}$ de $C_{\boldsymbol{G}^{*}}^{0}(s)$ ainsi qu'un tore maximal $F^{*}$-stable $\boldsymbol{T}_{1}^{*}$ de $\boldsymbol{B}_{1}^{*}$. On notera $W(s)$ (resp. $W^{0}(s)$ ) le groupe de Weyl de $C_{\boldsymbol{G}^{*}}(s)$ (resp. $\left.C_{\boldsymbol{G}^{*}}^{0}(s)\right)$ relatif à $\boldsymbol{T}_{1}^{*}$. Le groupe $A_{\boldsymbol{G}^{*}}(s)$ sera identifié au sous-groupe

$$
\left\{w \in W(s) \mid{ }^{w} \boldsymbol{B}_{1}^{*}=\boldsymbol{B}_{1}^{*}\right\}
$$

de $W(s)$. On a donc

$$
W(s)=W^{0}(s) \rtimes A_{\boldsymbol{G}^{*}}(s) .
$$

Si $w \in W^{0}(s)$, on notera $\boldsymbol{T}_{w}^{*}$ un tore maximal $F^{*}$-stable de $C_{\boldsymbol{G}^{*}}^{0}(s)$ de type $w$. On pose alors

$$
\chi_{s}^{\boldsymbol{G}}=\chi_{s}=\frac{\varepsilon_{\boldsymbol{G}} \varepsilon_{\boldsymbol{G}^{*}}(s)}{\left|W^{0}(s)\right|} \sum_{w \in W^{0}(s)} \varepsilon(w) R_{\boldsymbol{T}_{w}^{*}}^{\boldsymbol{G}}(s),
$$

où $\varepsilon: W^{0}(s) \rightarrow\{1,-1\}$ désigne la signature de $W^{0}(s)$ et $R_{\boldsymbol{T}_{w}^{*}}^{\boldsymbol{G}_{*}}(s)$ désigne le caractère de Deligne-Lusztig associé à la paire $\left(\boldsymbol{T}_{w}^{*}, s\right)$. On rappelle le résultat suivant ( $c f$. [A, prop 3.1.1 et 3.2.1] et [DLM, prop. 3.12]).

Proposition 3.2.3.

(a) Pour tout $a \in H^{1}(F, \boldsymbol{Z})$, on $a\left\langle\chi_{s}, \Gamma_{\psi_{a}}^{\boldsymbol{G}}\right\rangle_{\boldsymbol{G}^{F}}=1$.

(b) La fonction centrale $\chi_{s}$ sur $\boldsymbol{G}^{F}$ est en fait un caractère (non virtuel) de $\boldsymbol{G}^{F}$ sans multiplicité. De plus, $\chi_{s} \in \mathbb{Z} \mathcal{E}\left(\boldsymbol{G}^{F},[s]\right)$.

(c) Soit $\chi_{s, 1}$ la composante irréductible commune à $\chi_{s}$ et $\Gamma_{\psi}^{G}$. Si $\xi$ est un caractère linéaire de $A_{\boldsymbol{G}^{*}}(s)^{F^{*}}$, on choisit un élément $c \in H^{1}(F, \boldsymbol{Z})$ tel que $\widehat{\omega}_{s}^{0}(c)=\xi$ et on pose

$$
\chi_{s, \xi}=\tau_{c}^{\boldsymbol{G}} \chi_{s, 1} .
$$

Alors $\chi_{s, \xi}$ ne dépend pas du choix de c mais seulement de $\xi$ et

(d) Pour tout $c \in H^{1}(F, \boldsymbol{Z})$,

$$
\chi_{s}=\sum_{\xi \in\left(A_{G^{*}}(s)^{*}\right)^{\wedge}} \chi_{s, \xi}
$$

$$
\Gamma_{\psi_{c}}^{\boldsymbol{G}}=\sum_{[s]} \chi_{s, \widehat{\omega}_{s}^{0}(c)}
$$

où $[s]$ parcourt l'ensemble des classes de conjugaison d'éléments semi-simples de $\boldsymbol{G}^{* F^{*}}$. 
Corollaire 3.2.4. - Soit $\phi$ un caractère linéaire de $\boldsymbol{Z}^{F}$ et soit $c \in H^{1}(F, \boldsymbol{Z})$. Alors :

$$
\left\langle\chi_{s}, \Gamma_{\phi, \psi_{c}}^{\boldsymbol{G}}\right\rangle_{\boldsymbol{G}^{F}}= \begin{cases}1 & \text { si } \phi=\phi_{s} \\ 0 & \text { sinon }\end{cases}
$$

$$
\Gamma_{\phi, \psi_{c}}^{\boldsymbol{G}}=\sum_{[s] \mid \phi_{s}=\phi} \chi_{s, \widehat{\omega}_{s}^{0}(c)}
$$

Preuve. - Cela résulte de 3.1.1.

On posera, pour tout $(a, \zeta) \in \mathcal{M}^{\vee}\left(A_{\boldsymbol{G}^{*}}(s), F^{*}\right)$,

$$
\widehat{\chi}_{s,(a, \zeta)}^{\boldsymbol{G}}=\widehat{\chi}_{s,(a, \zeta)}=\sum_{(\alpha, \xi) \in \mathcal{M}\left(A_{\boldsymbol{G}^{*}}(s), F^{*}\right)}\{(\alpha, \xi),(a, \zeta)\} \chi_{s_{\alpha}, \xi}
$$

\section{Fonctions absolument cuspidales en type $\boldsymbol{A}$}

Dorénavant, et ce jusqu'à la fin de cet article, nous ferons l'hypothèse suivante :

$$
\boldsymbol{G} \text { est de type } A \text {. }
$$

Sous cette hypothèse, les fonctions absolument cuspidales ( $c f . \S 4.2$ pour la définition d'une fonction absolument cuspidale) sur $\boldsymbol{G}^{F}$ ont été déterminées dans [B2, cor. 6.2.2].

Nous allons rappeler ici comment construire une base explicite de $\operatorname{Cus}\left(\boldsymbol{G}^{F}\right)$. Nous allons même préciser le résultat de [B2] en exprimant les éléments de cette base comme combinaisons linéaires explicites de caractères irréductibles de $G^{F}$. Comme conséquence importante pour la suite, nous obtenons que l'opérateur de torsion stabilise les sous-espaces $\overline{\mathbb{Q}}_{\ell} \mathcal{E}\left(\boldsymbol{G}^{F},(s)\right)$, où $s$ est un élément semi-simple de $G^{* F^{*}}$.

4.1. Formule de Mackey. - D'après [B2, th. 5.2.1], la formule de Mackey pour les foncteurs de Lusztig a lieu dans $\boldsymbol{G}$. Cela implique que les foncteurs de Lusztig dans $\boldsymbol{G}$ ne dépendent pas du choix du sous-groupe parabolique. Par conséquent, si $\boldsymbol{L}$ est un sous-groupe de Levi $F$-stable d'un sous-groupe parabolique $\boldsymbol{P}$ de $\boldsymbol{G}$, on notera quelquefois $R_{\boldsymbol{L}}^{\boldsymbol{G}}$ et ${ }^{*} R_{\boldsymbol{L}}^{\boldsymbol{G}}$ les foncteurs de Lusztig $R_{\boldsymbol{L} \subset \boldsymbol{P}}^{\boldsymbol{G}}$ et ${ }^{*} R_{\boldsymbol{L} \subset \boldsymbol{P}}^{\boldsymbol{G}}$.

4.2. Fonctions absolument cuspidales. - On rappelle qu'une fonction centrale $\gamma$ sur $\boldsymbol{G}^{F}$ est dite absolument cuspidale si, pour tout sous-groupe de Levi rationnel $\boldsymbol{L}$ d'un sous-groupe parabolique $\boldsymbol{P}$ de $\boldsymbol{G}$, on a ${ }^{*} R_{\boldsymbol{L} \subset \boldsymbol{P}}^{\boldsymbol{G}} \gamma=0$. 
On notera $\operatorname{Cus}\left(\boldsymbol{G}^{F}\right)$ le sous-espace vectoriel de $\operatorname{Cent}\left(\boldsymbol{G}^{F}\right)$ formé des fonctions absolument cuspidales. L'espace vectoriel $\operatorname{Cus}\left(\boldsymbol{G}^{F}\right)$ a été déterminé dans [B2, cor. 6.2.2]. On va rappeler ici comment construire une base $\operatorname{de} \operatorname{Cus}\left(\boldsymbol{G}^{F}\right)$.

Soient $\phi$ et $\zeta$ des caractères linéaires de $\boldsymbol{Z}^{F}$ et $H^{1}(F, \boldsymbol{Z})$ respectivement. On pose :

$$
\dot{\Gamma}_{\phi, \zeta}^{\boldsymbol{G}}=\sum_{c \in H^{1}(F, \boldsymbol{Z})} \zeta(c) \Gamma_{\phi, \psi_{c}}^{\boldsymbol{G}} .
$$

THÉORÈme 4.2.1. - La famille $\left(\dot{\Gamma}_{\phi, \zeta}^{G}\right)_{\substack{\phi \in\left(\boldsymbol{Z}^{F}\right)^{\wedge} \\ \zeta \in H^{1}(F, \boldsymbol{Z})_{\text {cus }}^{\wedge}}}$ forme une base orthogonale de $\operatorname{Cus}\left(\boldsymbol{G}^{F}\right)$.

Preuve. - Soient $\phi$ et $\zeta$ deux caractères linéaires de $\boldsymbol{Z}^{F}$ et $H^{1}(F, \boldsymbol{Z})$ respectivement. Supposons $\zeta$ cuspidal. Si $c \in H^{1}(F, \boldsymbol{Z})$ et $z \in \boldsymbol{Z}^{F}$, alors

$$
\tau_{c}^{G} \dot{\Gamma}_{\phi, \zeta}^{G}=\zeta(c)^{-1} \dot{\Gamma}_{\phi, \zeta}^{G}, \quad t_{z}^{G} \dot{\Gamma}_{\phi, \zeta}^{G}=\phi(z) \dot{\Gamma}_{\phi, \zeta}^{G}
$$

Donc, d'après [B2, cor. 1.8.6], $\dot{\Gamma}_{\phi, \zeta}^{G}$ est une fonction absolument cuspidale et, d'après [B2, cor. 6.2.2, (a)], il suffit de montrer que $\dot{\Gamma}_{\phi, \zeta}^{G} \neq 0$. Pour cela, on va calculer son carré scalaire. Puisque $\dot{\Gamma}_{\phi, \zeta}^{G}$ est absolument cuspidale, on a $D_{G} \dot{\Gamma}_{\phi, \zeta}^{G}=\eta_{G} \dot{\Gamma}_{\phi, \zeta}^{G}$. En particulier, on a

$$
\left\langle\dot{\Gamma}_{\phi, \zeta}^{\boldsymbol{G}}, \dot{\Gamma}_{\phi, \zeta}^{\boldsymbol{G}}\right\rangle_{\boldsymbol{G}^{F}}=\eta_{\boldsymbol{G}}\left\langle D_{\boldsymbol{G}} \dot{\Gamma}_{\phi, \zeta}^{\boldsymbol{G}}, \dot{\Gamma}_{\phi, \zeta}^{\boldsymbol{G}}\right\rangle_{\boldsymbol{G}^{F}}
$$

Il résulte alors de la proposition 3.1 .3 que

$$
\left\langle\dot{\Gamma}_{\phi, \zeta}^{\boldsymbol{G}}, \dot{\Gamma}_{\phi, \zeta}^{\boldsymbol{G}}\right\rangle_{\boldsymbol{G}^{F}}=\left|H^{1}(F, \boldsymbol{Z})\right|
$$

Cela termine la démonstration.

Soit $(z, \zeta) \in \mathcal{M}^{\vee}\left(\boldsymbol{Z} / \boldsymbol{Z}^{0}, F\right)$. On pose

$$
\begin{aligned}
\widehat{\Gamma}_{\phi, \psi,(z, \zeta)}^{\boldsymbol{G}} & =\sum_{(c, \xi) \in \mathcal{M}\left(\boldsymbol{Z} / \boldsymbol{Z}^{0}, F\right)}\{(c, \xi),(z, \zeta)\} \Gamma_{\phi \xi, \psi_{c}}^{\boldsymbol{G}} \\
& =\frac{1}{\left|H^{1}(F, \boldsymbol{Z})\right|} \sum_{\xi \in\left(\boldsymbol{Z}^{F} / \boldsymbol{Z}^{\circ F}\right)^{\wedge}} \xi(z) \dot{\Gamma}_{\phi \xi, \zeta}^{\boldsymbol{G}} .
\end{aligned}
$$

4.3. Séries de Lusztig. - Dans ce paragraphe, nous allons préciser le théorème 4.2.1. Nous allons donner une base des espaces $\operatorname{Cus}\left(\boldsymbol{G}^{F}\right) \cap \overline{\mathbb{Q}}_{\ell} \mathcal{E}\left(\boldsymbol{G}^{F},[s]\right)$ et $\left.\operatorname{Cus}\left(\boldsymbol{G}^{F}\right) \cap \overline{\mathbb{Q}}_{\ell} \mathcal{E}\left(\boldsymbol{G}^{F},(s)\right)\right)$ pour $s$ un élément semi-simple de $\boldsymbol{G}^{* F^{*}}$ et nous

$$
\text { TOME } 128-2000-\mathrm{N}^{\circ} 3
$$


allons exprimer les éléments de cette base comme combinaisons linéaires explicites de caractères irréductibles appartenant à $\mathcal{E}\left(\boldsymbol{G}^{F},[s]\right)$ ou $\mathcal{E}\left(\boldsymbol{G}^{F},(s)\right)$. Avant toute chose, il convient de remarquer que

$$
\operatorname{Cus}\left(\boldsymbol{G}^{F}\right)=\bigoplus_{[s]}\left(\operatorname{Cus}\left(\boldsymbol{G}^{F}\right) \cap \overline{\mathbb{Q}}_{\ell} \mathcal{E}\left(\boldsymbol{G}^{F},[s]\right)\right)
$$

où $[s]$ parcourt l'ensemble des classes de conjugaison rationnelles d'éléments semi-simples de $G^{* F^{*}}$. De même, on a

$$
\operatorname{Cus}\left(\boldsymbol{G}^{F}\right)=\bigoplus_{(s)}\left(\operatorname{Cus}\left(\boldsymbol{G}^{F}\right) \cap \overline{\mathbb{Q}}_{\ell} \mathcal{E}\left(\boldsymbol{G}^{F},(s)\right)\right)
$$

où $(s)$ parcourt l'ensemble des classes de conjugaison $F^{*}$-stables d'éléments semisimples de $\boldsymbol{G}^{*}$.

ThÉORÈme 4.3.3. - Supposons $H^{1}(F, \boldsymbol{Z})_{\text {cus }}^{\wedge}$ non vide. Soit $\phi$ un caractère linéaire de $\boldsymbol{Z}^{F}$. Alors :

(a) Il existe un élément semi-simple $s \in G^{* F^{*}}$ tel que :

1) $s$ est rationnellement cuspidal;

2) $\phi_{s}=\phi$.

(b) Si s' est un élément semi-simple de $\boldsymbol{G}^{* F^{*}}$ vérifiant les propriétés 1) et 2) précédentes, alors $s^{\prime}$ est rationnellement conjugué à $s$.

(c) Soit $\zeta$ un caractère linéaire cuspidal de $H^{1}(F, \boldsymbol{Z})$. Puisque s est cuspidal, le morphisme de groupes $\omega_{s}^{0}: A_{\boldsymbol{G}^{*}}(s)^{F^{*}} \rightarrow H^{1}(F, \boldsymbol{Z})^{\wedge}$ est un isomorphisme (cf. prop. 1.4.9). Si a désigne l'élément de $A_{\boldsymbol{G}^{*}}(s)^{F^{*}}$ tel que $\omega_{s}^{0}(a)=\zeta$, alors

$$
\dot{\Gamma}_{\phi_{s}, \zeta}^{G}=\sum_{\xi \in\left(A_{G^{*}}(s)^{F^{*}}\right)^{\wedge}} \xi(a) \chi_{s, \xi}
$$

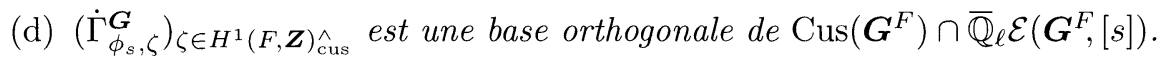

Preuve. - Soit $\zeta$ un caractère linéaire cuspidal de $H^{1}(F, \boldsymbol{Z})$. D'après [B2, cor. 6.2.2, (b)], il existe un unique (à $G^{* F^{*}}$-conjugaison près) élément semisimple $s \in \boldsymbol{G}^{* F^{*}}$ tel que $\dot{\Gamma}_{\phi, \zeta}^{G} \in \overline{\mathbb{Q}}_{\ell} \mathcal{E}\left(\boldsymbol{G}^{F},[s]\right)$. En particulier, $\phi_{s}=\phi$. Puisque $\tau_{c}^{\boldsymbol{G}} \dot{\Gamma}_{\phi, \zeta}^{\boldsymbol{G}}=\zeta(c)^{-1} \dot{\Gamma}_{\phi, \zeta}^{\boldsymbol{G}}$ pour tout $c \in H^{1}(F, \boldsymbol{Z})$, le noyau de $\zeta$ est contenu dans le noyau de $\widehat{\omega}_{s}^{0}\left(c f\right.$. [B2, 2.8.7]), c'est-à-dire que $\zeta$ est dans l'image de $\omega_{s}^{0}$. Par conséquent, $s$ est rationnellement cuspidal : cela montre le (a) du théorème 4.3.3.

Montrons maintenant le (c). D'après la proposition $1.4 .9, \omega_{s}: A_{G^{*}}(s) \rightarrow$ $\left(\boldsymbol{Z} / \boldsymbol{Z}^{0}\right)^{\wedge}$ est un isomorphisme donc les morphismes $\omega_{s}^{0}, \omega_{s}^{1}, \widehat{\omega}_{s}, \widehat{\omega}_{s}^{0}$ et $\widehat{\omega}_{s}^{1}$ sont aussi des isomorphismes. Soit $a$ l'unique élément de $A_{\boldsymbol{G}^{*}}(s)^{F^{*}}$ tel que $\omega_{s}^{0}(a)=\zeta$. Posons

$$
\Gamma=\sum_{\xi \in\left(A_{G^{*}}(s)^{F^{*}}\right)^{\wedge}} \xi(a) \chi_{s, \xi}
$$

BULLETIN DE LA SOCIÉTÉ MATHÉMATIQUE DE FRANCE 
Alors $\Gamma$ est une fonction centrale sur $\boldsymbol{G}^{F}$ et on a, pour tout $z \in \boldsymbol{Z}^{F}$ et $c \in H^{1}(F, \boldsymbol{Z})$,

$$
t_{z}^{G} \Gamma=\phi(z) \Gamma, \quad \tau_{c}^{G} \Gamma=\zeta(c)^{-1} \Gamma .
$$

La première égalité résulte de 0.4 .2 et la deuxième résulte de la proposition 3.2.3, (c). D'après [B2, cor. 6.2.2, (a)], les fonctions $\Gamma$ et $\dot{\Gamma}_{\phi, \zeta}^{G}$ sont proportionnelles. Le résultat découle alors du fait que

$$
\left\langle\dot{\Gamma}_{\phi, \zeta}^{\boldsymbol{G}}, \chi_{s, 1}\right\rangle_{\boldsymbol{G}^{F}}=1=\left\langle\Gamma, \chi_{s, 1}\right\rangle_{\boldsymbol{G}^{F}}
$$

la première égalité résultant du corollaire 3.2 .4 , (b), et du fait que $\omega_{s}^{0}$ est un isomorphisme.

Le (b) et le (d) résultent du (c) et de 0.4.2.

ThÉorème 4.3.4. - Supposons $H^{1}(F, \boldsymbol{Z})_{\mathrm{cus}}^{\wedge}$ non vide. Soit $\phi^{0}$ un caractère linéaire de $\boldsymbol{Z}^{0 F}$.

(a) Il existe un élément semi-simple $s$ de $G^{* F^{*}}$ vérifiant les propriétés suivantes :

1) $s$ est cuspidal;

2) $\operatorname{Res} \boldsymbol{Z}^{\boldsymbol{Z}^{\mathrm{F}}} \phi_{s}=\phi^{0}$.

(b) Si $s^{\prime}$ est un autre élément semi-simple de $\boldsymbol{G}^{* F^{*}}$ vérifiant les conditions 1) et 2) ci-dessus, alors $s^{\prime}$ est géométriquement conjugué à $s$.

(c) Soit $(z, \zeta) \in \mathcal{M}^{\vee}\left(\boldsymbol{Z} / \boldsymbol{Z}^{0}, F\right)$. On suppose $\zeta$ cuspidal. Puisque $\omega_{s}$ est un isomorphisme (cf. proposition 1.4.9), il existe $(a, \sigma)$ dans $\mathcal{M}^{\vee}\left(A_{G^{*}}(s), F^{*}\right)$ tel que $\omega_{s}^{0}(a)=\zeta$ et $\widehat{\omega}_{s}^{1}(z)=\sigma$. Alors

$$
\widehat{\Gamma}_{\phi_{s}, \psi,(z, \zeta)}^{G}=\widehat{\chi}_{s,(a, \sigma)} .
$$

(d) $\left(\widehat{\Gamma}_{\phi_{s}, \psi,(z, \zeta)}^{\boldsymbol{G}}\right)_{\substack{z \in \boldsymbol{Z}^{F} / \boldsymbol{z}^{\circ} \boldsymbol{F} \\ \zeta \in H^{1}(F, \boldsymbol{Z}) \hat{\mathrm{cus}}}}$ est une base orthonormale de

$$
\operatorname{Cus}\left(\boldsymbol{G}^{F}\right) \cap \overline{\mathbb{Q}}_{\ell} \mathcal{E}\left(\boldsymbol{G}^{F},(s)\right) .
$$

Preuve. - Soit $\phi$ un caractère linéaire de $Z^{F}$ étendant $\phi^{0}$. Soit $s$ l'unique (à $\boldsymbol{G}^{* F^{*}}$-conjugaison près) élément semi-simple cuspidal de $\boldsymbol{G}^{* F^{*}}$ tel que $\phi_{s}=\phi$ (cf. th. 4.3.3). Alors $s$ satisfait les conditions (1) et (2) du (a). En particulier, d'après 1.4.9, le morphisme $\omega_{s}: A_{\boldsymbol{G}^{*}}(s) \rightarrow\left(\boldsymbol{Z} / \boldsymbol{Z}^{0}\right)^{\wedge}$ est un isomorphisme. Donc, si $\phi^{\prime}$ est un autre caractère linéaire de $Z^{F}$ étendant $\phi^{0}$, alors il existe $\alpha \in H^{1}\left(F^{*}, A_{G^{*}}(s)\right)$ tel que $\phi_{s_{\alpha}}=\phi^{\prime}$ (cf. lemme 1.3.4). Le (d) découle de cette remarque et du théorème 4.3 .3 , partie $(\mathrm{d})$.

La partie (b) découle $\mathrm{du}(\mathrm{d})$ et la partie (c) résulte du théorème 4.3.3, (c) et du lemme 1.3.4.

TOME $128-2000-\mathrm{N}^{\circ} 3$ 
Corollaire 4.3.5. - Soit s un élément semi-simple de $\boldsymbol{G}^{* F^{*}}$. Alors l'opérateur de torsion $\operatorname{Sh}_{F / F}^{G}$ stabilise $\overline{\mathbb{Q}}_{\ell} \mathcal{E}\left(\boldsymbol{G}^{F},(s)\right)$.

Preuve. - D'après le $(\mathrm{d})$ du théorème 4.3 .4 et le corollaire 2.2.5, $\mathrm{Sh}_{F / F}^{G}$ stabilise $\operatorname{Cus}\left(\boldsymbol{G}^{F}\right) \cap \overline{\mathbb{Q}}_{\ell} \mathcal{E}\left(\boldsymbol{G}^{F},(s)\right)$.

Soit maintenant $\gamma \in \overline{\mathbb{Q}}_{\ell} \mathcal{E}\left(\boldsymbol{G}^{F},(s)\right)$. Il existe une famille $\mathcal{A}$ de paires $(\boldsymbol{L}, \lambda)$ telles que $\boldsymbol{L}$ est un sous-groupe de Levi $F$-stable d'un sous-groupe parabolique de $\boldsymbol{G}, \lambda$ est une fonction absolument cuspidale sur $\boldsymbol{L}^{F}$ telles que

$$
\gamma=\sum_{(\boldsymbol{L}, \lambda) \in \mathcal{A}} R_{\boldsymbol{L}}^{\boldsymbol{G}} \lambda
$$

D'autre part, d'après [B1, cor. 4.4.1], on peut supposer que $\lambda \in \mathcal{E}\left(\boldsymbol{L}^{F},\left(t_{\boldsymbol{L}}\right)_{\boldsymbol{L}^{*}}\right)$ où $\boldsymbol{L}^{*}$ est un sous-groupe de Levi $F^{*}$-stable d'un sous-groupe parabolique de $\boldsymbol{G}^{*}$ dual de $\boldsymbol{L}$ et $t_{\boldsymbol{L}}$ est un élément semi-simple de $\boldsymbol{L}^{* F^{*}}$ géométriquement conjugué à $s((\boldsymbol{L}, \lambda) \in \mathcal{A})$. De plus, d'après [DM1, 2.2], on a

$$
\mathrm{Sh}_{F / F}^{G} \circ R_{L}^{G}=R_{L}^{G} \circ \operatorname{Sh}_{F / F}^{L} .
$$

Par conséquent,

$$
\operatorname{Sh}_{F / F}^{G} \gamma=\sum_{(\boldsymbol{L}, \lambda) \in \mathcal{A}} R_{\boldsymbol{L}}^{\boldsymbol{G}}\left(\operatorname{Sh}_{F / F}^{\boldsymbol{L}} \lambda\right) .
$$

Or, si $(\boldsymbol{L}, \lambda) \in \mathcal{A}$, on a $\operatorname{Sh}_{F / F}^{\boldsymbol{L}} \lambda \in \overline{\mathbb{Q}}_{\ell} \mathcal{E}\left(\boldsymbol{L}^{F},\left(t_{\boldsymbol{L}}\right)_{\boldsymbol{L}^{*}}\right)$ car $\lambda$ est absolument cuspidale donc $\operatorname{Sh}_{F / F}^{\boldsymbol{G}} \gamma \in \overline{\mathbb{Q}}_{\ell} \mathcal{E}\left(\boldsymbol{G}^{F},(s)_{\boldsymbol{G}^{*}}\right)$ d'après [B1, cor. 4.4.1].

\section{Opérateur de torsion dans $G^{F}$}

Dans cette partie, on se fixe un élément semi-simple $s$ de $G^{* F^{*}}$ et on note $\tilde{s}$ un élément semi-simple de $\widetilde{G}^{* F^{*}}$ tel que $i^{*}(\tilde{s})=s$. On reprend toutes les notations du paragraphe $3.2\left(\boldsymbol{B}_{1}^{*}, \boldsymbol{T}_{w}^{*}, \chi_{s, \xi}, s_{\alpha} \ldots\right)$. On posera

$$
\widetilde{\boldsymbol{B}}_{1}^{*}=i^{*-1}\left(\boldsymbol{B}_{1}^{*}\right), \quad \widetilde{\boldsymbol{T}}_{1}^{*}=i^{*-1}\left(\boldsymbol{T}_{1}^{*}\right) .
$$

Le groupe $W^{0}(s)$ s'identifie avec le groupe $W(\tilde{s})$ (groupe de Weyl de $C_{\widetilde{G}^{*}}(\tilde{s})$ relatif à $\left.\widetilde{\boldsymbol{T}}_{1}^{*}\right)$ car $C_{\widetilde{\boldsymbol{G}}^{*}}(\tilde{s})$ est connexe. Pour tout $w \in W(\tilde{s})$, on posera

$$
\widetilde{\boldsymbol{T}}_{w}^{*}=i^{*-1}\left(\boldsymbol{T}_{w}^{*}\right)
$$

5.1. Caractères irréductibles de $\widetilde{\boldsymbol{G}}^{\boldsymbol{F}}$. - Soit $\chi$ un caractère irréductible $F^{*}$-stable de $W(\tilde{s})$. D'après [LS, th. 3.2], il existe une unique extension $\tilde{\chi}$ de $\chi$ à $W(\tilde{s}) \rtimes\left\langle F^{*}\right\rangle$ telle que la fonction centrale

$$
R_{\chi}^{\widetilde{\boldsymbol{G}}}[\tilde{s}]=\frac{{ }^{\varepsilon} \widetilde{\boldsymbol{G}}^{\varepsilon} C_{\widetilde{\boldsymbol{G}}^{*}(\tilde{s})}}{|W(\tilde{s})|} \sum_{w \in W(\tilde{s})} \tilde{\chi}\left(w F^{*}\right) R_{\widetilde{\boldsymbol{T}}_{w}^{*}}^{\widetilde{\boldsymbol{G}}_{w}}(\tilde{s})
$$


soit un caractère irréductible de $\widetilde{\boldsymbol{G}}^{F}$. De plus, d'après [LS, th. 3.2], l'application

$$
\begin{aligned}
(\operatorname{Irr} W(\tilde{s}))^{F^{*}} & \longrightarrow \mathcal{E}\left(\widetilde{\boldsymbol{G}}^{F},[\tilde{s}]\right) \\
\chi & \longmapsto R_{\chi}^{\widetilde{\boldsymbol{G}}}[\tilde{s}]
\end{aligned}
$$

est bijective.

5.2. Caractères de Gel'fand-Graev généralisés. - Soit $u$ un élément unipotent de $G^{F}$. Pour les groupes de type $A$, on dispose, quelle que soit la caractéristique du corps sur lequel on travaille, d'une théorie de DynkinKostant pour classifier les classes unipotentes. En particulier, on peut associer canoniquement à $u$ un sous-groupe parabolique $F$-stable $\widetilde{\boldsymbol{P}}(u)$ de $\widetilde{\boldsymbol{G}}$ dont on notera $\boldsymbol{U}(u)$ le radical unipotent. On fixera aussi un sous-groupe de Levi $F$ stable $\widetilde{\boldsymbol{L}}(u)$ de $\widetilde{\boldsymbol{P}}(u)$. On pose :

$$
\boldsymbol{P}(u)=\widetilde{\boldsymbol{P}}(u) \cap \boldsymbol{G}, \quad \boldsymbol{L}(u)=\widetilde{\boldsymbol{L}}(u) \cap \boldsymbol{G} .
$$

Ce sous-groupe parabolique vérifie entre autres les propriétés suivantes :

1) $u$ appartient à $\boldsymbol{U}(u)$;

2) $C_{\widetilde{\boldsymbol{G}}}(u)$ est contenu dans $\widetilde{\boldsymbol{P}}(u)$.

Dans [K2], N. Kawanaka a aussi associé à $u$ un caractère irréductible $\lambda^{u}$ de $\boldsymbol{U}(u)^{F}$ et posé la définition suivante :

$$
\Gamma_{u}^{G}=\operatorname{Ind} \underset{U(u)^{F}}{G^{F}} \lambda^{u}
$$

Le caractère $\boldsymbol{\Gamma}_{u}^{\boldsymbol{G}}$ est appelé caractère de Gel'fand-Graev généralisé de $\boldsymbol{G}^{F}$. Il ne dépend que de la classe de conjugaison de $u$ dans $G^{F}$. Lorsque $u$ est un élément unipotent régulier, on retrouve les caractères de Gel'fand-Graev classiques.

On note Uni $(\boldsymbol{G})$ l'ensemble des classes unipotentes de $\boldsymbol{G}$. Pour chaque classe unipotente $C \in \operatorname{Uni}(\boldsymbol{G})^{F}$, on fixe un élément unipotent $F$-stable $u_{C}$. Dans $\widetilde{\boldsymbol{G}}$, les centralisateurs d'éléments sont connexes, donc $C^{F}$ est égal à $\left[u_{C}\right]_{\widetilde{G}^{F}}$. D'autre part, G. Lusztig [L1, 13.4] a construit une application

$$
\begin{aligned}
\operatorname{Irr} \widetilde{\boldsymbol{G}}^{F} & \longrightarrow \operatorname{Uni}(\widetilde{\boldsymbol{G}})^{F} \\
\gamma & \longmapsto C_{\gamma} .
\end{aligned}
$$

Dans le cas des groupes de type $A$ à centre connexe, cette application vérifie la propriété suivante ( $c f$. [K1, th. 3.2.11, cor. 3.2.18 et rem. 3.2.24, (i)] ; il faut aussi utiliser le fait que les fonctions de Green sont des polynômes en $q$ ) :

TOME $128-2000-\mathrm{N}^{\circ} 3$ 
ThÉORÈme 5.2.2 (Kawanaka). - Soit $\gamma$ un caractère irréductible de $\widetilde{\boldsymbol{G}}^{F}$ et soit $C$ une classe unipotente $F$-stable de $\widetilde{\boldsymbol{G}}$. Alors

$$
\left\langle\boldsymbol{\Gamma}_{u_{C}}^{\widetilde{\boldsymbol{G}}}, \gamma\right\rangle_{\widetilde{\boldsymbol{G}}^{F}}= \begin{cases}1 & \text { si } C_{\gamma}=C, \\ 0 & \text { si } C \text { n'est pas contenu dans } \bar{C}_{\gamma} .\end{cases}
$$

Si $\chi$ est un caractère irréductible $F^{*}$-stable de $W(\tilde{s})$, on notera $C_{s, \chi}$ la classe de conjugaison $C_{\gamma}$, où $\gamma=R_{\chi}^{\widetilde{G}}[\tilde{s}]$.

5.3. Caractères irréductibles de $\boldsymbol{G}^{\boldsymbol{F}}$. - Si $\chi$ est un caractère irréductible $F^{*}$-stable de $W(\tilde{s})=W^{0}(s)$, alors on pose

$$
R_{\chi}^{\boldsymbol{G}}[s]=\frac{\varepsilon_{\boldsymbol{G}^{\varepsilon}} C_{\boldsymbol{G}^{*}}(s)}{\left|W^{0}(s)\right|} \sum_{w \in W^{0}(s)} \tilde{\chi}\left(w F^{*}\right) R_{\boldsymbol{T}_{w}^{*}}^{\boldsymbol{G}}(s) .
$$

Il résulte de [B1, prop. 4.3.3, (i)] que

$$
R_{\chi}^{\boldsymbol{G}}[s]=\operatorname{Res} \underset{\boldsymbol{G}^{F}}{\widetilde{\boldsymbol{G}}^{F}} R_{\chi}^{\widetilde{\boldsymbol{G}}}[\tilde{s}] .
$$

Grâce au principe de réciprocité de Frobenius, on déduit du théorème 5.2.2 que

$$
\left\langle\boldsymbol{\Gamma}_{u_{C}}^{\boldsymbol{G}}, R_{\chi}^{\boldsymbol{G}}[s]\right\rangle_{\widetilde{\boldsymbol{G}}^{F}}= \begin{cases}1 & \text { si } C_{s, \chi}=C, \\ 0 & \text { si } C \text { n'est pas contenu dans } \bar{C}_{s, \chi} .\end{cases}
$$

En particulier, le caractère $R_{\chi}^{G}[s]$ est sans multiplicité car toutes ses composantes irréductibles ont même multiplicité par la théorie de Clifford, et on notera $R_{\chi}^{G}[s]_{1}$ la composante irréductible commune à $R_{\chi}^{G}[s]$ et $\Gamma_{u_{C_{s, \chi}}}^{G}$. Pour décomposer complètement le caractère $R_{\chi}^{G}[s]$, il ne reste plus qu'à calculer le stabilisateur de $R_{\chi}^{G}[s]_{1}$ dans $\widetilde{\boldsymbol{G}}^{F}$. Tout d'abord, ce stabilisateur contient $\widetilde{\boldsymbol{Z}}^{F}$. Notons $A_{\boldsymbol{G}^{*}}(s, \chi)$ le stabilisateur de $\chi$ dans $A_{\boldsymbol{G}^{*}}(s)$ et notons $\widetilde{\boldsymbol{G}}^{F}(s, \chi)$ le noyau du morphisme composé

$$
\widetilde{\boldsymbol{G}}^{F} \longrightarrow \widetilde{\boldsymbol{G}}^{F} / \boldsymbol{G}^{F} . \widetilde{\boldsymbol{Z}}^{F} \stackrel{\sigma_{\boldsymbol{G}}}{\longrightarrow} H^{1}(F, \boldsymbol{Z}) \stackrel{\widehat{\omega}_{s}^{0}}{\longrightarrow}\left(A_{\boldsymbol{G}^{*}}(s)^{F^{*}}\right)^{\wedge} \longrightarrow\left(A_{\boldsymbol{G}^{*}}(s, \chi)^{F^{*}}\right)^{\wedge}
$$

Proposition 5.3.3. - Le stablisateur de $R_{\chi}^{\boldsymbol{G}}[s]_{1}$ dans $\widetilde{\boldsymbol{G}}^{F}$ est $\widetilde{\boldsymbol{G}}^{F}(s, \chi)$.

Preuve. - Cela résulte de la théorie de Clifford et du fait que

$$
R_{\widetilde{\boldsymbol{T}}_{w}^{*}}^{\widetilde{\boldsymbol{G}}_{w}}(\tilde{s}) \otimes \boldsymbol{\omega}_{\boldsymbol{G}, F}\left(\widetilde{\omega}_{s}(a)\right)=R_{\widetilde{\boldsymbol{T}}_{a}^{*}-1 w a}^{\widetilde{G}_{w a}}(\tilde{s})
$$

pour tous $w \in W^{\circ}(s)$ et $a \in A_{\boldsymbol{G}^{*}}(s)^{F^{*}}$. $\square$ 
Si $\xi$ est un caractère linéaire de $A_{G^{*}}(s, \chi)^{F^{*}}$, on notera $\tilde{g}_{\xi}$ un élément de $\widetilde{\boldsymbol{G}}^{F}$ dont l'image par le morphisme composé ci-dessus est $\xi$ et on posera

$$
R_{\chi}^{G}[s]_{\xi}=R_{\chi}^{G}[s]_{1} \circ\left(\operatorname{int} \tilde{g}_{\xi}\right)^{-1} .
$$

Il est facile de vérifier que, pour tous $c \in H^{1}(F, Z)$ et $\xi \in\left(A_{G^{*}}(s, \chi)^{F^{*}}\right)^{\wedge}$, on a

$$
\tau_{c}^{G} R_{\chi}^{G}[s]_{\xi}=R_{\chi}^{G}[s]_{\xi \xi_{c, \chi}},
$$

où $\xi_{c, \chi}=\operatorname{Res}{ }_{A_{G^{*}}(s, \chi)^{F^{*}}}^{A_{G^{*}}} \widehat{\omega}_{s}^{0}(c)$.

On notera $\mathcal{I}\left(W^{0}(s), A_{G^{*}}(s), F^{*}\right)$ l'ensemble des paires $(\chi, \xi)$ où $\chi$ est un caractère irréductible $F^{*}$-stable de $W^{0}(s)$ et $\xi$ est un caractère linéaire de $A_{G^{*}}(s, \chi)^{F^{*}}$.

Proposition 5.3.6.

(a) L'application suivante est surjective:

$$
\begin{aligned}
\mathcal{I}\left(W^{0}(s), A_{\boldsymbol{G}^{*}}(s), F^{*}\right) & \longrightarrow \mathcal{E}\left(\boldsymbol{G}^{F},[s]\right), \\
(\chi, \xi) & \longmapsto R_{\chi}^{\boldsymbol{G}}[s]_{\xi} .
\end{aligned}
$$

(b) Soient $(\chi, \xi)$ et $\left(\chi^{\prime}, \xi^{\prime}\right)$ deux éléments de $\mathcal{I}\left(W^{0}(s), A_{G^{*}}(s), F^{*}\right)$. Alors $R_{\chi}^{G}[s]_{\xi}=R_{\chi^{\prime}}^{G}[s]_{\xi^{\prime}}$ si et seulement si il existe $a \in A_{G^{*}}(s)^{F^{*}}$ tel que $\left(\chi^{\prime}, \xi^{\prime}\right)=$ ${ }^{a}(\chi, \xi)$ ou, de manière équivalente puisque $A_{\boldsymbol{G}^{*}}(s)$ est abélien, $\left(\chi^{\prime}, \xi^{\prime}\right)=\left({ }^{a} \chi, \xi\right)$.

Preuve. - Cela résulte de la théorie de Clifford et de 5.3.4.

5.4. Familles de $\boldsymbol{G}^{\boldsymbol{F}}$. - Avant d'énoncer le théorème principal de cet article, nous allons décomposer la série de Lusztig géométrique $\mathcal{E}\left(\boldsymbol{G}^{F},(s)\right)$ en familles de la même manière que dans [Sh3, 4.4]. Le raisonnement que nous allons suivre est déjà contenu dans [Sh3, 4.4] mais, puisqu'il s'applique à une classe plus grande de groupes réductifs finis, nous allons le rappeler. Tout d'abord, on a

$$
\mathcal{E}\left(\boldsymbol{G}^{F},(s)\right)=\coprod_{\alpha \in H^{1}\left(F^{*}, A_{\boldsymbol{G}^{*}}(s)\right)} \mathcal{E}\left(\boldsymbol{G}^{F},\left[s_{\alpha}\right]\right) .
$$

Pour tout $\alpha \in H^{1}\left(F^{*}, A_{\boldsymbol{G}^{*}}(s)\right)$, on notera $\delta(\alpha)$ l'élément de $A_{\boldsymbol{G}^{*}}(s)$ dont $g_{\alpha}^{-1} F\left(g_{\alpha}\right)$ est un représentant dans $C_{\boldsymbol{G}^{*}}(s)$. Alors le groupe $W\left(s_{\alpha}\right)$ peut-être identifié au groupe $W(s)$ muni du morphisme de Frobenius $\delta(\alpha) F^{*}$.

Soit $\chi$ un caractère irréductible de $W^{0}(s)$. S'il existe un élément $\alpha \in$ $H^{1}\left(F^{*}, A_{\boldsymbol{G}^{*}}(s)\right)$ tel que $\chi$ soit $\delta(\alpha) F^{*}$-stable, alors l'orbite de $\chi$ sous $A_{\boldsymbol{G}^{*}}(s)$ est $F^{*}$-stable. Réciproquement, si l'orbite de $\chi$ sous $A_{G^{*}}(s)$ est $F^{*}$-stable, alors il existe un élément $\alpha \in H^{1}\left(F^{*}, A_{G^{*}}(s)\right)$ et un élément $a \in A_{G^{*}}(s)$ tels que $\chi={ }^{a^{-1} \delta(\alpha) F^{*}(a)}\left(F^{*} \chi\right)$. Il est alors facile de vérifier que ${ }^{a} \chi$ est $\delta(\alpha) F^{*}$-stable. 
Cela montre qu'il faut s'intéresser à l'ensemble des orbites $F^{*}$-stables de caractères irréductibles de $W^{0}(s)$ sous l'action de $A_{G^{*}}(s)$.

On notera $A_{\boldsymbol{G}^{*}}(s) \backslash \operatorname{Irr} W^{0}(s)$ l'ensemble des orbites de $A_{\boldsymbol{G}^{*}}(s)$ dans $\operatorname{Irr} W^{0}(s)$. Si $\chi$ est un caractère irréductible de $W^{0}(s)$, on notera $[\chi]$ son orbite sous l'action de $A_{G^{*}}(s)$.

Fixons un élément $F^{*}$-stable $[\chi] \in A_{G^{*}}(s) \backslash \operatorname{Irr} W^{0}(s)$. Le calcul précédent montre que l'on peut supposer que $\chi$ est $\delta\left(\alpha_{\chi}\right) F^{*}$-stable pour un $\alpha_{\chi} \in$ $H^{1}\left(F^{*}, A_{G^{*}}(s)\right)$ : il est à noter que la paire $\left(\chi, \alpha_{\chi}\right)$ n'est pas déterminée de manière unique. L'élément $\alpha_{\chi}$ n'est même pas déterminé par le choix particulier de $\chi$. Notons

$$
\mu_{\chi}: H^{1}\left(F^{*}, A_{\boldsymbol{G}^{*}}(s, \chi)\right) \longrightarrow H^{1}\left(F^{*}, A_{\boldsymbol{G}^{*}}(s)\right)
$$

le morphisme naturel de groupes.

Soit $(\alpha, \xi) \in \mathcal{M}\left(A_{G^{*}}(s, \chi), F^{*}\right)$. Alors il existe un élément $a \in A_{G^{*}}(s)$ tel que

$$
a^{-1} \delta\left(\mu_{\chi}(\alpha) \alpha_{\chi}\right) \delta\left(\alpha_{\chi}\right)^{-1} F^{*}(a)
$$

appartienne à $A_{G^{*}}(s, \chi)$ et représente $\alpha$. On pose alors

$$
\chi_{\alpha}={ }^{a} \chi .
$$

Le caractère $\chi_{\alpha}$ est déterminé à conjugaison près par un élément de $A_{G^{*}}(s)^{F^{*}}$ et il est facile de vérifier que $\chi_{\alpha}$ est $\delta\left(\mu_{\chi}(\alpha) \alpha_{\chi}\right) F^{*}$-stable. On peut donc alors poser :

$$
R_{\lceil\chi]}^{G}(s)_{\alpha, \xi}=R_{\chi_{\alpha}}^{G}\left[s_{\mu_{\chi}(\alpha) \alpha_{\chi}}\right]_{\xi} .
$$

Le caractère $R_{[\chi]}^{G}(s)_{\alpha, \xi}$ dépend alors seulement du choix de $\chi$ et $\alpha_{\chi}$.

La proposition suivante est prouvée dans [Sh3,4.4] dans un cas particulier : la preuve s'applique mot à mot à la situation plus générale de cet article.

Proposition 5.4.2. - Soient $(\alpha, \xi)$ et $\left(\alpha^{\prime}, \xi^{\prime}\right)$ deux éléments de l'ensemble $\mathcal{M}\left(A_{G^{*}}(s, \chi), F^{*}\right)$. Alors $R_{[\chi]}^{\boldsymbol{G}}(s)_{\alpha, \xi}=R_{[\chi]}^{\boldsymbol{G}}(s)_{\alpha^{\prime}, \xi^{\prime}}$ si et seulement si $(\alpha, \xi)=$ $\left(\alpha^{\prime}, \xi^{\prime}\right)$.

On pose :

$$
\mathcal{F}_{[\chi]}\left(\boldsymbol{G}^{F},(s)\right)=\left\{R_{[\chi]}^{\boldsymbol{G}}(s)_{\alpha, \xi} \mid(\alpha, \xi) \in \mathcal{M}\left(A_{\boldsymbol{G}^{*}}(s, \chi), F^{*}\right)\right\} .
$$

Alors l'ensemble $\mathcal{F}_{[\chi]}\left(\boldsymbol{G}^{F},(s)\right)$ dépend seulement de $[\chi]$ et non du choix de $\chi$ et $\alpha_{\chi}$ et il est facile de vérifier que

$$
\mathcal{E}\left(\boldsymbol{G}^{F},(s)\right)=\coprod_{[\chi] \in\left(A_{\boldsymbol{G}^{*}}(s) \backslash \operatorname{Irr} W^{0}(s)\right)^{F^{*}}} \mathcal{F}_{[\chi]}\left(\boldsymbol{G}^{F},(s)\right) .
$$

De plus, la proposition 5.4.2 montre que l'on a une bijection

$$
\mathcal{M}\left(A_{G^{*}}(s, \chi), F^{*}\right) \stackrel{\sim}{\longrightarrow} \mathcal{F}_{[\chi]}\left(G^{F},(s)\right)
$$

pour tout $[\chi] \in\left(A_{G^{*}}(s) \backslash \operatorname{Irr} W^{0}(s)\right)^{F^{*}}$. 
5.5. Caractères fantômes. - Soit maintenant $(a, \sigma)$ un élément de $\mathcal{M}^{\vee}\left(A_{G^{*}}(s, \chi), F^{*}\right)$. On pose :

$$
\widehat{R}_{[\chi]}^{\boldsymbol{G}}(s)_{a, \sigma}=\sum_{(\alpha, \xi) \in \mathcal{M}\left(A_{\boldsymbol{G}^{*}}(s, \chi), F^{*}\right)}\{(\alpha, \xi),(a, \sigma)\} R_{[\chi]}^{\boldsymbol{G}}(s)_{\alpha, \xi}
$$

On appelle $\widehat{R}_{[\chi]}^{\boldsymbol{G}}(s)_{a, \sigma}$ un caractère fantôme de $\boldsymbol{G}^{F}$.

Si $(\alpha, \xi) \in \mathcal{M}\left(A_{\boldsymbol{G}^{*}}(s, \chi), F^{*}\right)$, on a

$$
R_{[\chi]}^{\boldsymbol{G}}(s)_{\alpha, \xi}=\sum_{(a, \sigma) \in \mathcal{M}^{\vee}\left(A_{G^{*}}(s, \chi), F^{*}\right)} \overline{\{(\alpha, \xi),(a, \sigma)\}} \widehat{R}_{[\chi]}^{\boldsymbol{G}}(s)_{a, \sigma} .
$$

La proposition suivante est immédiate :

Proposition 5.5.3. - $\left(\widehat{R}_{[\chi]}^{G}(s)_{a, \sigma}\right)_{(a, \sigma) \in \mathcal{M}^{\vee}\left(A_{G^{*}}(s, \chi), F^{*}\right)}$ est une base orthonormale de l'espace vectoriel $\overline{\mathbb{Q}}_{\ell} \mathcal{F}_{[\chi]}\left(\boldsymbol{G}^{F},(s)\right)$.

Remarque. - La bijection 5.4 .4 dépend de beaucoup de choses : elle dépend du choix d'un représentant $F$-stable dans chaque classe de conjugaison unipotente $F$-stable de $\boldsymbol{G}$, du choix d'un représentant $F^{*}$-stable $s$ de la classe de conjugaison géométrique $(s)$, du choix de $\chi$ dans $[\chi]$ et du choix de $\alpha_{\chi}$. Cependant, si on effectue d'autres choix à chacune de ces étapes, le caractère fantôme $\widehat{R}_{[\chi]}^{\boldsymbol{G}}(s)_{a, \sigma}\left((a, \sigma) \in \mathcal{M}^{\vee}\left(A_{\boldsymbol{G}^{*}}(s, \chi), F^{*}\right)\right)$ ne sera modifié que par une racine de l'unité. Cela donne plus de sens à la définition de caractère fantôme.

Le théorème suivant s'en trouve aussi conforté :

ThÉORÈme 5.5.4. - Soient $\chi$ et $\alpha_{\chi}$ comme ci-dessus. On suppose que $F$ agit trivialement sur $\boldsymbol{Z} / \boldsymbol{Z}^{0}$. Soit $(a, \sigma) \in \mathcal{M}^{\vee}\left(A_{G^{*}}(s, \chi), F^{*}\right)$. Alors

$$
\operatorname{Sh}_{F / F}^{G} \widehat{R}_{[\chi]}^{G}(s)_{a, \sigma}=\sigma(\bar{a})^{-1} \widehat{R}_{[\chi]}^{G}(s)_{a, \sigma}
$$

où $\bar{a}$ désigne la classe de a dans $H^{1}\left(F^{*}, A_{G^{*}}(s, \chi)\right)$.

La fin de cette partie est consacrée à la preuve du théorème 5.5.4.

5.6. Réduction du problème. - Puisque $F$ agit trivialement sur $\boldsymbol{Z} / \boldsymbol{Z}^{0}$ et puisque $\omega_{s}$ commute avec l'action des morphismes de Frobenius $F$ et $F^{*}$, le morphisme $F^{*}$ agit trivialement sur $A_{G^{*}}(s)$. En particulier, on peut identifier les morphismes $\omega_{s}, \omega_{s}^{0}$ et $\omega_{s}^{1}$, ainsi que les morphismes $\widehat{\omega}_{s}$, $\widehat{\omega}_{s}^{0}$ et $\widehat{\omega}_{s}^{1}$. D'autre part, la section $\delta: H^{1}\left(F^{*}, A_{\boldsymbol{G}^{*}}(s)\right) \rightarrow A_{\boldsymbol{G}^{*}}(s)$ est égale à l'identité.

$$
\text { TOME } 128-2000-\mathrm{N}^{\circ} 3
$$


Soit donc $[\chi] \in\left(A_{G^{*}}(s) \backslash \operatorname{Irr} W^{0}(s)\right)^{F^{*}}$ et soient $\chi$ et $\alpha_{\chi}$ comme précédemment. Comme cela est expliqué dans la remarque ci-dessus, on peut, pour prouver le théorème 5.5.4, changer beaucoup des choix qui ont été faits plus haut. En particulier, en remplaçant $s$ par $s_{\alpha_{\chi}}$, on peut supposer que $\alpha_{\chi}=1$, de sorte que $\chi$ est $F^{*}$-stable. Par ailleurs, l'application $\operatorname{Irr} \boldsymbol{G}^{F} \rightarrow \operatorname{Uni}\left(\boldsymbol{G}^{F}\right)$ est constante sur la famille $\mathcal{F}_{[\chi]}\left(\boldsymbol{G}^{F},(s)\right)$, donc on notera $C_{\chi}$ la classe de conjugaison unipotente $F$-stable associée à tous les caractères irréductibles de $\mathcal{F}_{[\chi]}\left(\boldsymbol{G}^{F},(s)\right)$.

Remarquons aussi que

$$
\mathcal{M}\left(A_{\boldsymbol{G}^{*}}(s, \chi), F^{*}\right)=\mathcal{M}^{\vee}\left(A_{\boldsymbol{G}^{*}}(s, \chi), F^{*}\right)=A_{\boldsymbol{G}^{*}}(s, \chi) \times A_{\boldsymbol{G}^{*}}(s, \chi)^{\wedge} .
$$

On définit la relation d'ordre partiel $\preccurlyeq$ sur l'ensemble Uni $(\boldsymbol{G})^{F}$ de la manière suivante. Soient $C$ et $C^{\prime}$ deux classes unipotentes $F$-stables de $\boldsymbol{G}$. Alors

$$
C \preccurlyeq C^{\prime} \text { si et seulement si } C \subset \bar{C}^{\prime} \text {. }
$$

On écrira $C \prec C^{\prime}$ pour dire que l'on a $C \preccurlyeq C^{\prime}$ et $C \neq C^{\prime}$.

On va raisonner par récurrence sur la dimension de $\boldsymbol{G}$ et aussi par récurrence, mais descendante, sur l'ensemble $\operatorname{Uni}(\boldsymbol{G})^{F}$ muni de l'ordre partiel $\preccurlyeq$. On supposera donc que le théorème 5.5.4 est vrai dans tout sous-groupe de Levi $F$-stable d'un sous-groupe parabolique propre de $\boldsymbol{G}$, et qu'il est aussi vrai pour toute famille $\mathcal{F}_{\left[\chi^{\prime}\right]}\left(\boldsymbol{G}^{F},(s)\right)$ telle que $C_{\chi} \prec C_{\chi^{\prime}}$.

5.7. Sous-groupes de Levi et foncteurs de Lusztig. - Soit $\boldsymbol{L}^{*}$ un sousgroupe de Levi $F^{*}$-stable d'un sous-groupe parabolique de $\boldsymbol{G}^{*}$ et soit $\boldsymbol{L}$ un sous-groupe de Levi $F$-stable d'un sous-groupe parabolique $\boldsymbol{P}$ de $\boldsymbol{G}$ dual de $\boldsymbol{L}^{*}$. On note $\boldsymbol{U}$ le radical unipotent de $\boldsymbol{P}$ et on pose

$$
\boldsymbol{Y}_{\boldsymbol{U}}^{\boldsymbol{G}}=\left\{g \in \boldsymbol{G} \mid g^{-1} F(g) \in \boldsymbol{U}\right\} .
$$

On notera $H_{c}^{i}\left(\boldsymbol{Y}_{U}^{\boldsymbol{G}}\right)$ le $i$-ième groupe de cohomologie à support compact à coefficients dans le faisceau constant $\overline{\mathbb{Q}}_{\ell}$.

Puisque $\boldsymbol{G}^{F}$ et $\boldsymbol{L}^{F}$ agissent sur $\boldsymbol{Y}_{\boldsymbol{U}}^{\boldsymbol{G}}$ par translations à gauche et à droite respectivement, l'espace vectoriel $H_{c}^{i}\left(\boldsymbol{Y}_{\boldsymbol{U}}^{G}\right)$ hérite d'une structure de $\boldsymbol{G}^{F}$-module$\boldsymbol{L}^{F}$. La preuve du théorème suivant est disponible dans une version corrigée de [DM3, th. 13.25] :

Théorème 5.7.1. - Supposons que $C_{\boldsymbol{G}^{*}}\left(\right.$ s) soit contenu dans $\boldsymbol{L}^{*}$. Alors:

(a) Soit $\lambda \in \mathcal{E}\left(\boldsymbol{L}^{F},[s]_{\boldsymbol{L}^{* F^{*}}}\right)$. Il existe un entier naturel $i(\lambda)$ tel que $H_{c}^{i}\left(\boldsymbol{Y}_{\boldsymbol{U}}^{\boldsymbol{G}}\right) \otimes_{\overline{\mathbb{Q}}_{\ell} \boldsymbol{L}^{F}} \lambda=0$ si $i \neq i(\lambda)$ et tel que le $\boldsymbol{G}^{F}$-module $H_{c}^{i(\lambda)}\left(\boldsymbol{Y}_{\boldsymbol{U}}^{\boldsymbol{G}}\right) \otimes_{\overline{\mathbb{Q}}_{\ell} \boldsymbol{L}^{F}} \lambda$ soit irréductible. De plus, $(-1)^{i(\lambda)}=\varepsilon_{\boldsymbol{G}} \varepsilon_{\boldsymbol{L}}$.

(b) L'application $\varepsilon_{\boldsymbol{G} \varepsilon_{\boldsymbol{L}}} R_{\boldsymbol{L} \subset \boldsymbol{P}}^{\boldsymbol{G}}: \mathcal{E}\left(\boldsymbol{G}^{F},[s]_{\boldsymbol{L}^{* F^{*}}}\right) \rightarrow \mathcal{E}\left(\boldsymbol{G}^{F},[s]_{\boldsymbol{G}^{* F^{*}}}\right)$ est bijective. 
Corollaire 5.7.2. - Supposons que $C_{\boldsymbol{G}^{*}}^{0}\left(\right.$ s) soit contenu dans $\boldsymbol{L}^{*}$. Soit $\lambda \in$ $\mathcal{E}\left(\boldsymbol{L}^{F},[s]_{\boldsymbol{L}^{* F^{*}}}\right)$. Alors il existe un entier naturel $i(\lambda)$ tel que $H_{c}^{i}\left(\boldsymbol{Y}_{\boldsymbol{U}}^{\boldsymbol{G}}\right) \otimes_{\overline{\mathbb{Q}}_{\ell} \boldsymbol{L}^{F}} \lambda=0$

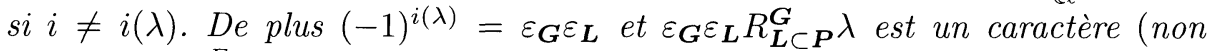
virtuel) de $\boldsymbol{G}^{F}$.

Preuve. - Notons $\widetilde{\boldsymbol{L}}=\boldsymbol{L} . \widetilde{\boldsymbol{Z}}$ et $\widetilde{\boldsymbol{L}}^{*}=i^{*-1}\left(\boldsymbol{L}^{*}\right)$. Remarquons tout d'abord que $\widetilde{\boldsymbol{L}}^{*}$ contient $C_{\widetilde{\boldsymbol{G}}^{*}}(\tilde{s})$ car ce dernier est connexe et a pour image dans $\boldsymbol{G}^{*}$ le groupe $C_{\boldsymbol{G}^{*}}^{0}(s)$. D'après [B1, lemme 4.3.6], il existe $\tilde{\lambda} \in \mathcal{E}\left(\widetilde{\boldsymbol{G}}^{F},[\tilde{s}]_{\widetilde{\boldsymbol{L}}^{* F^{*}}}\right)$ tel que $\lambda$ soit une composante irréductible de la restriction de $\tilde{\lambda}$ à $\boldsymbol{L}^{F}$. D'après [B1, 4.3.1 et 4.3.2], le $\boldsymbol{G}^{F}$-module $H_{c}^{i}\left(\boldsymbol{Y}_{\boldsymbol{U}}^{\boldsymbol{G}}\right) \otimes_{\overline{\mathbb{Q}}_{\ell} \boldsymbol{L}^{F}} \lambda$ est un sous-module de la restriction à $\boldsymbol{G}^{F} \mathrm{du}$ $\widetilde{\boldsymbol{G}}^{F}$-module $H_{c}^{i}\left(\boldsymbol{Y}_{\boldsymbol{U}}^{\widetilde{G}}\right) \otimes_{\overline{\mathbb{Q}}_{\ell} \widetilde{\boldsymbol{L}}^{F}} \tilde{\lambda}$. Le corollaire 5.7.2 résulte alors du théorème 5.7.1 appliqué au groupe $\widetilde{\boldsymbol{G}}$ : il suffit de prendre $i(\lambda)=i(\tilde{\lambda})$.

REMARQUE.-Le théorème 5.7.1 et le corollaire 5.7.2 sont vrais dans n'importe quel groupe réductif, sans hypothèse sur l'action de $F$ sur $\boldsymbol{Z} / \boldsymbol{Z}^{0}$. Le fait que $\boldsymbol{G}$ soit de type $A$ n'intervient pas dans la démonstration et l'action de $F$ non plus. Cependant, le corollaire suivant utilise que $\boldsymbol{G}$ est de type $A$, puisqu'il utilise le paramétrage des caractères irréductibles de $\boldsymbol{G}^{F}$, et il utilise que $F$ agit trivialement sur $\boldsymbol{Z} / \boldsymbol{Z}^{0}$.

Corollaire 5.7.3. - Notons $C_{\boldsymbol{G}^{*}}(s, \chi)$ l'image inverse de $A_{\boldsymbol{G}^{*}}(s, \chi)$ dans $C_{\boldsymbol{G}^{*}}(s)$ par le morphisme canonique $C_{\boldsymbol{G}^{*}}(s) \rightarrow A_{\boldsymbol{G}^{*}}(s)$ et supposons que $\boldsymbol{L}^{*}$ contienne $C_{\boldsymbol{G}^{*}}(s, \chi)$. Alors $\varepsilon_{\boldsymbol{G}} \boldsymbol{\varepsilon}_{\boldsymbol{L}} R_{\boldsymbol{L} \subset \boldsymbol{P}}^{\boldsymbol{G}}$ induit une bijection $\mathcal{F}_{[\chi]}\left(\boldsymbol{L}^{F},(s)_{\boldsymbol{L}^{*}}\right) \rightarrow$ $\mathcal{F}_{[\chi]}\left(\boldsymbol{G}^{F},(s)_{\boldsymbol{G}^{*}}\right)$.

Preuve. - Remarquons tout d'abord que $A_{\boldsymbol{L}^{*}}(s, \chi)=A_{\boldsymbol{G}^{*}}(s, \chi)$ donc $\left|\mathcal{F}_{[\chi]}\left(\boldsymbol{L}^{F},(s)_{\boldsymbol{L}^{*}}\right)\right|=\left|\mathcal{F}_{[\chi]}\left(\boldsymbol{G}^{F},(s)_{\boldsymbol{G}^{*}}\right)\right|$.

Soit $(\alpha, \xi) \in \mathcal{M}\left(A_{\boldsymbol{L}^{*}}(s, \chi), F^{*}\right)$. Si on note $s_{\alpha}^{\boldsymbol{L}}$ l'élément de $\boldsymbol{L}^{* F^{*}}$ dont la classe de $\boldsymbol{L}^{* F^{*}}$-conjugaison est associée à $\alpha$, alors $s_{\alpha}^{\boldsymbol{L}}$ est $\boldsymbol{G}^{* F^{*}}$-conjugué à $s_{\alpha}$ car $F^{*}$ agit trivialement sur $A_{G^{*}}(s)$. En particulier, si $\left(\alpha^{\prime}, \xi^{\prime}\right) \in \mathcal{M}\left(A_{L^{*}}(s, \chi), F^{*}\right)$ est tel que

$$
\varepsilon_{\boldsymbol{G}} \varepsilon_{\boldsymbol{L}} R_{\boldsymbol{L} \subset \boldsymbol{P}}^{\boldsymbol{G}} R_{[\chi]}^{\boldsymbol{L}}(s)_{\alpha, \xi}=\varepsilon_{\boldsymbol{G}} \varepsilon_{\boldsymbol{L}} R_{\boldsymbol{L} \subset \boldsymbol{P}}^{\boldsymbol{G}} R_{[\chi]}^{\boldsymbol{L}}(s)_{\alpha^{\prime}, \xi^{\prime}},
$$

alors on a $\alpha=\alpha^{\prime}$. Donc, pour montrer le corollaire 5.7.3, il suffit alors de remarquer que $R_{\chi}^{\boldsymbol{L}}\left[s_{\alpha}^{\boldsymbol{L}}\right]$ et $R_{\chi}^{\boldsymbol{G}}\left[s_{\alpha}\right]=\varepsilon_{\boldsymbol{G}} \varepsilon_{\boldsymbol{L}} R_{\boldsymbol{L} \subset \boldsymbol{P}}^{\boldsymbol{G}} R_{\chi}^{\boldsymbol{L}}\left[s_{\alpha}^{\boldsymbol{L}}\right]$ ont le même nombre de composantes irréductibles et d'utiliser le corollaire 5.7.2.

Nous terminons ce paragraphe par deux résultats à propos des centralisateurs d'éléments semi-simples dans les groupes de type $A$.

TOME $128-2000-\mathrm{N}^{\circ} 3$ 
Lemme 5.7.4. - Soit $\boldsymbol{H}^{*}$ un sous-groupe fermé $F^{*}$-stable propre de $C_{\boldsymbol{G}^{*}}(s)$ contenant $C_{\boldsymbol{G}^{*}}^{0}(s)$. Alors il existe un sous-groupe de Levi $F^{*}$-stable $\boldsymbol{L}^{*}$ d'un sousgroupe parabolique propre de $\boldsymbol{G}^{*}$ contenant $\boldsymbol{H}^{*}$.

Preuve. - Il suffit de montrer qu'il existe un sous-groupe de Levi d'un sous-groupe parabolique propre de $\boldsymbol{G}^{*}$ contenant $\boldsymbol{H}^{*}$. Pour obtenir un sousgroupe de Levi rationnel, il suffira de prendre l'intersection de tous ceux qui contiennent $\boldsymbol{H}^{*}$. L'application $A_{G^{*}}(s) \rightarrow A_{\widehat{G}^{*}}(\hat{s})$ est injective (où $\hat{s}=\pi^{*}(s)$ ), donc on peut se ramener au cas où $\boldsymbol{G}$ est semi-simple, simplement connexe et quasi-simple, c'est-à-dire $G=\mathrm{SL}_{n}(\mathbb{F})$ pour un entier naturel non nul $n$. On peut alors supposer que $\boldsymbol{G}^{*}=\mathrm{PGL}_{n}(\mathbb{F})$.

Supposons que $\boldsymbol{H}^{*}$ n'est contenu dans aucun sous-groupe de Levi d'un sousgroupe parabolique propre de $\boldsymbol{G}^{*}$. Puisque $\boldsymbol{H}^{*}$ est contenu dans le normalisateur de $C_{\boldsymbol{G}^{*}}^{0}(s)$ et contient $C_{\boldsymbol{G}^{*}}^{0}(s)$, cela implique qu'il existe un diviseur $d$ de $n$ (on pose $n=d k)$ tel que $s$ soit conjugué à $\operatorname{diag}\left(a_{1}, \ldots, a_{1}, a_{2}, \ldots, a_{2}, \ldots, a_{k}, \ldots, a_{k}\right)$ où $a_{1}, \ldots, a_{k}$ sont des éléments de $\mathbb{F}^{\times}$deux à deux distincts apparaissant chacun $d$ fois dans l'écriture précédente.

Le groupe $A_{G^{*}}(s)$ est isomorphe, via $\widetilde{\omega}_{s}$, au sous-groupe $A$ de $\mathbb{F}^{\times}$défini comme suit :

$$
A=\left\{x \in \mathbb{F}^{\times} \mid\left\{x a_{1}, \ldots, x a_{k}\right\}=\left\{a_{1}, \ldots, a_{k}\right\}\right\} .
$$

Notons $A^{\prime}$ le sous-groupe de $A$ image de $\boldsymbol{H}^{*}$. Si $\left|A^{\prime}\right|=k^{\prime}$, et si $\Omega$ désigne une orbite de $A^{\prime}$ dans $\left\{a_{1}, \ldots, a_{k}\right\}$, on peut supposer que $\Omega=\left\{a_{1}, \ldots, a_{k^{\prime}}\right\}$ ce qui montre que $\boldsymbol{H}^{*}$ est contenu dans l'image de $\mathrm{GL}_{d k^{\prime}}(\mathbb{F}) \times \mathrm{GL}_{d\left(n-k^{\prime}\right)}(\mathbb{F})$ dans $\mathrm{PGL}_{n}(\mathbb{F})$. Puisque $\boldsymbol{H}^{*}$ n'est contenu dans aucun sous-groupe de Levi d'un sousgroupe parabolique propre de $G^{*}$, cela implique que $k^{\prime}=k$ donc que $A^{\prime}=A$, c'est-à-dire $\boldsymbol{H}^{*}=C_{\boldsymbol{G}^{*}}(s)$.

L'élément semi-simple $s$ est dit quasi-isolé si $C_{\boldsymbol{G}^{*}}(s)$ n'est contenu dans aucun sous-groupe de Levi d'un sous-groupe parabolique propre de $\boldsymbol{G}^{*}$.

Lemme 5.7.5. - Supposons s quasi-isolé et soient $\chi$ et $\chi^{\prime}$ deux caractères irréductibles de $W^{0}(s)$ invariants sous $A_{G^{*}}(s)$ et tels que $C_{\chi}=C_{\chi^{\prime}}$. Alors $\chi=\chi^{\prime}$.

Preuve. - Utilisons à nouveau le morphisme $\pi^{*}: \boldsymbol{G}^{*} \rightarrow \widehat{\boldsymbol{G}}^{*}$. Alors $\hat{s}=\pi^{*}(s)$ est quasi-isolé dans $\widehat{\boldsymbol{G}}^{*}$ et, d'après le lemme 5.7.4, l'application $C_{\boldsymbol{G}^{*}}(s) \rightarrow C_{\widehat{\boldsymbol{G}}^{*}}(\hat{s})$ est surjective. En particulier, cela montre que l'on peut encore supposer que $G^{*}=\mathrm{PGL}_{n}(\mathbb{F})$.

Par suite, il existe un diviseur $d$ de $n$, premier à $p$, et tel que $s$ soit conjugué à l'image $\operatorname{diag}\left(1, \zeta, \ldots, \zeta^{d-1}\right) \otimes I_{k}$ dans $\mathrm{PGL}_{n}(\mathbb{F})$ où $n=d k$ et $I_{k}$ désigne la matrice identité de $\mathrm{GL}_{k}(\mathbb{F})$. Dans ce cas, $W^{0}(s)$ est isomorphe à $\left(\mathfrak{S}_{k}\right)^{d}$ où $\mathfrak{S}_{k}$ désigne le groupe symétrique de degré $k$, et $A_{G^{*}}(s)$ est le groupe cyclique d'ordre $d$, agissant sur $\left(\mathfrak{S}_{k}\right)^{d}$ par permutation transitive des composantes. 
Un caractère irréductible $\chi$ de $W^{0}(s)$ invariant sous $A_{G^{*}}(s)$ est donc de la forme $\chi_{1} \otimes \cdots \otimes \chi_{1}$ où $\chi_{1}$ est un caractère irréductible de $\mathfrak{S}_{k}$. Notons $\mu$ la partition de $k$ associé à $\chi_{1}$. Alors la classe unipotente de $\boldsymbol{G}$ associé à $\chi$ est la classe paramétrée par la partition de $n$ duale de $d \mu$. Cela montre le lemme.

5.8. Fin de la preuve du théorème 5.5.4. - Soit $u=u_{C_{s, \chi}}$. Si $\phi$ est un caractère linéaire de $\boldsymbol{Z}^{F}$, on pose :

$$
\Gamma_{\phi, u}^{G}=\operatorname{Ind}_{\boldsymbol{Z}^{F} \times \boldsymbol{U}(u)^{F}}^{\boldsymbol{G}^{F}}\left(\phi \otimes \lambda^{u}\right) .
$$

D'autre part, si $(z, \zeta) \in \mathcal{M}^{\vee}\left(\boldsymbol{Z} / \boldsymbol{Z}^{0}, F\right)$, on pose

$$
\widehat{\boldsymbol{\Gamma}}_{\phi, u,(z, \zeta)}^{G}=\sum_{(c, \xi) \in \mathcal{M}\left(\boldsymbol{Z} / \boldsymbol{Z}^{0}, F\right)}\{(c, \xi),(z, \zeta)\} \tau_{c}^{G} \boldsymbol{\Gamma}_{\phi \xi, u}^{G} .
$$

D'après la proposition 2.2 .5 , on a

$$
\operatorname{Sh}_{F / F}^{G} \widehat{\boldsymbol{\Gamma}}_{\phi, u,(z, \zeta)}^{G}=\zeta(\bar{z})^{-1} \widehat{\boldsymbol{\Gamma}}_{\phi, u,(z, \zeta)}^{G} .
$$

Soit $(a, \sigma) \in \mathcal{M}^{\vee}\left(A_{G^{*}}(s, \chi), F^{*}\right)$. On pose $\zeta=\omega_{s}^{1}(a)$ et soit $z$ un élément de $\boldsymbol{Z}^{F}$ dont l'image par le morphisme composé

$$
\boldsymbol{Z}^{F} \longrightarrow \boldsymbol{Z}^{F} / \boldsymbol{Z}^{0 F} \stackrel{\widehat{\omega}_{s}^{1}}{\longrightarrow} H^{1}\left(F^{*}, A_{\boldsymbol{G}^{*}}(s)\right)^{\wedge} \longrightarrow H^{1}\left(F^{*}, A_{\boldsymbol{G}^{*}}(s, \chi)\right)^{\wedge}
$$

est égal à $\sigma$ (un tel $z$ existe car $F$ agit trivialement sur $\left.\boldsymbol{Z} / \boldsymbol{Z}^{0}\right)$. Alors $\sigma(\bar{a})=\zeta(\bar{z})$ où $\bar{z}$ est la classe de $z$ dans $H^{1}(F, \boldsymbol{Z})$.

Lemme 5.8.2. - Avec les notations ci-dessus, la projection de $\widehat{\boldsymbol{\Gamma}}_{\phi_{s}, u,(z, \zeta)}^{\boldsymbol{G}}$ sur $\overline{\mathbb{Q}}_{\ell} \mathcal{F}_{[\chi]}\left(\boldsymbol{G}^{F},(s)\right)$ est égale à $\widehat{R}_{[\chi]}^{\boldsymbol{G}}(s)_{a, \sigma}$.

Preuve. - Soit $(c, \xi) \in \mathcal{M}\left(\boldsymbol{Z} / \boldsymbol{Z}^{0}, F\right)$. On note $\xi^{\prime}=\operatorname{Res}_{A_{G^{*}}(s, \chi)}^{A_{G^{*}}(s, \chi)} \widehat{\omega}_{s}^{0}(c)$. Puisque

$$
t_{z}^{\boldsymbol{G}}\left(\tau_{c}^{\boldsymbol{G}} \boldsymbol{\Gamma}_{\phi_{s} \xi, u}^{\boldsymbol{G}}\right)=\phi_{x} \tau_{c}^{\boldsymbol{G}} \xi(z) \boldsymbol{\Gamma}_{\phi_{s} \xi, u}^{\boldsymbol{G}}
$$

pour tout $z \in \boldsymbol{Z}^{F}$, la projection de $\tau_{c}^{G} \boldsymbol{\Gamma}_{\phi_{s} \xi, u}^{\boldsymbol{G}}$ sur $\overline{\mathbb{Q}}_{\ell} \mathcal{F}_{[\chi]}\left(\boldsymbol{G}^{F},(s)\right)$ est égale à la projection de $\tau_{c}^{G} \boldsymbol{\Gamma}_{\phi_{s} \xi, u}^{\boldsymbol{G}}$ sur $\overline{\mathbb{Q}}_{\ell} \mathcal{F}_{[\chi]}\left(\boldsymbol{G}^{F},(s)\right) \cap \overline{\mathbb{Q}}_{\ell} \mathcal{E}\left(\boldsymbol{G}^{F},\left[s_{\alpha}\right]\right)$ si $\xi=\omega_{s}^{1}(\alpha)$ pour un $\alpha \in A_{G^{*}}(s, \chi)$ et est nulle si $\xi$ n'est pas dans l'image de $A_{G^{*}}(s, \chi)$ sous le morphisme $\omega_{s}^{1}$ (cela résulte de l'injectivité du morphisme $\omega_{s}^{1}$ et du lemme 1.3.4). Donc, si $\xi=\omega_{s}^{1}(\alpha)$ pour un $\alpha \in A_{G^{*}}(s, \chi)$, alors la projection de $\tau_{c}^{G} \boldsymbol{\Gamma}_{\phi_{s} \xi, u}^{G}$ $\operatorname{sur} \overline{\mathbb{Q}}_{\ell} \mathcal{F}_{[\chi]}\left(\boldsymbol{G}^{F},(s)\right)$ est égale à $R_{\chi}\left[s_{\alpha}\right]_{\xi^{\prime}}$. Le lemme 5.8.2 en découle.

Compte tenu de l'égalité 5.8.1 et du lemme 5.8.2, il suffit, pour montrer le théorème 5.5.4, de montrer que $\operatorname{Sh}_{F / F}^{G} \widehat{R}_{[\chi]}^{G}(s)_{a, \sigma}$ appartient à l'espace vectoriel TOME $128-2000-\mathrm{N}^{\circ} 3$ 
$\overline{\mathbb{Q}}_{\ell} \mathcal{F}_{[\chi]}\left(\boldsymbol{G}^{F},(s)\right)$. Compte tenu du corollaire 5.7.3, du lemme 5.7.4 et de l'hypothèse de récurrence, on peut supposer que $s$ est quasi-isolé dans $G^{*}$ et que $A_{\boldsymbol{G}^{*}}(s)=A_{\boldsymbol{G}^{*}}(s, \chi)$. Dans ce cas-là, la projection de $\widehat{\boldsymbol{\Gamma}}_{\phi_{s}, u,(z, \zeta)}^{\boldsymbol{G}} \operatorname{sur} \overline{\mathbb{Q}}_{\ell} \mathcal{E}\left(\boldsymbol{G}^{F},(s)\right)$ est égale à $\widehat{R}_{[\chi]}^{\boldsymbol{G}}(s)_{a, \sigma}$ plus des termes appartenant à des familles $\mathcal{F}_{\left[\chi^{\prime}\right]}\left(\boldsymbol{G}^{F},(s)\right)$, où $\left[\chi^{\prime}\right]$ est tel que $C_{\chi} \prec C_{\chi^{\prime}}$ d'après le lemme 5.7.5. Puisque $\operatorname{Sh}_{F / F}^{G}$ commute à la projection sur $\overline{\mathbb{Q}}_{\ell} \mathcal{E}\left(\boldsymbol{G}^{F},(s)\right)$ d'après le lemme 4.3.5, et puisque, par hypothèse de récurrence, les espaces vectoriels $\overline{\mathbb{Q}}_{\ell} \mathcal{F}_{\left[\chi^{\prime}\right]}\left(\boldsymbol{G}^{F},(s)\right)$ sont stables sous $\operatorname{Sh}_{F / F}^{G}$ (où $\chi^{\prime}$ est tel que $C_{\chi} \prec C_{\chi^{\prime}}$ ), le théorème 5.5.4 est démontré.

\section{Faisceaux-caractères củspidaux sur $G$}

On appellera système local un $\overline{\mathbb{Q}}_{\ell}$-système local.

6.1. Paires cuspidales. - Soit $C$ une classe de conjugaison quelconque de $\boldsymbol{G}$ et soit $\Sigma=\boldsymbol{Z}^{0} . C$. Soit $\mathcal{E}$ un système local irréductible $\boldsymbol{G}$-équivariant sur $\Sigma$. Puisque $\boldsymbol{G}$ est de type $A$, il résulte de [L2, prop. 2.7] que, si $\mathcal{E}$ est cuspidal au sens de [L2, déf. 2.4], alors $C=z C^{\prime}$, où $z \in Z$ et $C^{\prime}$ est une classe unipotente de $\boldsymbol{G}$. De plus, compte tenu de [L2, prop. 2.8], $C^{\prime}$ est alors la classe des unipotents réguliers de $\boldsymbol{G}$. Puisque la caractéristique $p$ de $\mathbb{F}$ est bonne pour $\boldsymbol{G}$, la classification des systèmes locaux irréductibles cuspidaux sur la classe des unipotents réguliers est donnée par la proposition 1.2 .2 et par la remarque suivant 1.2.1 (en effet, puisque $\boldsymbol{G}$ est de type $A$, tous les nombres premiers sont bons pour $\boldsymbol{G}$ ) : l'ensemble des classes d'isomorphismes est en bijection avec $\left(\boldsymbol{Z} / \boldsymbol{Z}^{0}\right)_{\text {cus }}^{\wedge}$. Ces quelques remarques vont permettre de classifier facilement les paires cuspidales $(\Sigma, \mathcal{E})$ cuspidales.

On reprend les notations du paragraphe 1.2. Par exemple, $C_{\text {reg }}$ désigne la classe des unipotents réguliers de $\boldsymbol{G}$. Soit $z \in Z$ et soit $\zeta$ un caractère linéaire cuspidal de $\boldsymbol{Z} / \boldsymbol{Z}^{0}$. On rappelle que $\mathcal{L}_{\zeta}$ désigne le système local irréductible

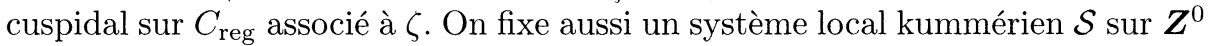
et on note $\mathcal{E}_{\mathcal{S}, z, \zeta}$ le système local irréductible

$$
T(z)_{*}\left(\mathcal{S} \otimes \mathcal{L}_{\zeta}\right) \simeq\left(T(z)_{*} \mathcal{S}\right) \otimes \mathcal{L}_{\zeta}
$$

sur $z \boldsymbol{Z}^{0} . C_{\text {reg }}$, où $T(z): \boldsymbol{G} \rightarrow \boldsymbol{G}, g \mapsto z g$ désigne la translation par $z$.

La proposition suivante résulte des remarques précédentes.

Proposition 6.1.1. - Soit $(\Sigma, \mathcal{E})$ une paire formée d'une sous-variété $\Sigma$ de $\boldsymbol{G}$ de la forme $\boldsymbol{Z}^{0} . C$, où $C$ est une classe de conjugaison de $\boldsymbol{G}$, et $\mathcal{E}$ est un système local irréductible $G$-équivariant sur $\Sigma$. Alors la paire $(\Sigma, \mathcal{E})$ est cuspidale si et seulement si il existe un élément $z$ de $\boldsymbol{Z}$, un caractère linéaire cuspidal $\zeta$ de $\boldsymbol{Z} / \boldsymbol{Z}^{0}$ et un système local kummérien $\mathcal{S}$ sur $\boldsymbol{Z}^{0}$ tels que $(\Sigma, \mathcal{E})=\left(z \boldsymbol{Z}^{0} . C_{\mathrm{reg}}, \mathcal{E}_{\mathcal{L}, z, \zeta}\right)$. 
Si $(\mathcal{S}, z, \zeta)$ sont comme ci-dessus, alors $T\left(z^{0}\right)_{*} \mathcal{S} \simeq \mathcal{S}$ pour tout $z^{0} \in Z^{0}$. Cela implique la proposition suivante :

Proposition 6.1.2. - Soient $(\mathcal{S}, z, \zeta)$ et $\left(\mathcal{S}^{\prime}, z^{\prime}, \zeta^{\prime}\right)$ deux triplets comme ci-dessus. Alors les paires $\left(z \boldsymbol{Z}^{0} . C_{\mathrm{reg}}, \mathcal{E}_{\mathcal{S}, z, \zeta}\right)$ et $\left(z^{\prime} \boldsymbol{Z}^{0} . C_{\mathrm{reg}}, \mathcal{E}_{\mathcal{S}^{\prime}, z^{\prime}, \zeta^{\prime}}\right)$ sont isomorphes si et seulement si $\mathcal{S} \simeq \mathcal{S}^{\prime}, \zeta=\zeta^{\prime}$ et $z^{\prime} z^{-1} \in Z^{0}$.

Corollaire 6.1.3. - La paire $\left(z \boldsymbol{Z}^{0} . C_{\mathrm{reg}}, \mathcal{E}_{\mathcal{S}, z, \zeta}\right)$ est $F$-stable si et seulement si $F^{*} \mathcal{S} \simeq \mathcal{S}, \zeta \in H^{1}(F, Z)_{\text {cus }}^{\wedge}$ et $z^{-1} F(z) \in Z^{0}$.

L'application $\boldsymbol{Z}^{0} \rightarrow \boldsymbol{Z}^{0}, z \mapsto z^{-1} F(z)$ est un revêtement étale galoisien de $\boldsymbol{Z}^{0}$ de groupe $\boldsymbol{Z}^{0 F}$. Si $\phi^{0}$ est un caractère linéaire de $\boldsymbol{Z}^{0 F}$, on notera $\mathcal{S}_{\phi^{0}}$ un système local kummérien sur $\boldsymbol{Z}^{0}$ associé à ce revêtement et au caractère linéaire $\phi^{0}$. Alors $\mathcal{S}_{\phi^{0}}$ est $F$-stable et tout système local kummérien $F$-stable sur $Z^{0}$ est isomorphe à un et un seul de ces $\mathcal{S}_{\phi^{0}}$.

On note $\mathcal{C U S}(\boldsymbol{G}, F)$ l'ensemble des triplets $\left(\phi^{0}, z, \zeta\right)$ où $\phi^{0}$ est un caractère linéaire de $\boldsymbol{Z}^{0 F}, z \in \boldsymbol{Z}^{F} / \boldsymbol{Z}^{0 F}$ et $\zeta \in H^{1}(F, \boldsymbol{Z})_{\text {cus }}^{\wedge}$. Si $\left(\phi^{0}, z, \zeta\right) \in \mathcal{C U S}(\boldsymbol{G}, F)$, on notera $\dot{z}$ un représentant de $z$ dans $\boldsymbol{Z}^{F}$ et on notera $\mathcal{E}_{\phi^{0}, z, \zeta}$ le système local $\mathcal{E}_{\mathcal{S}_{\phi^{0}}, \dot{z}, \zeta}$ sur $\dot{z} \boldsymbol{Z}^{0} . C_{\text {reg. }}$. Les paires $\left(\dot{z} \boldsymbol{Z}^{0} . C_{\text {reg }}, \mathcal{E}_{\phi^{0}, z, \zeta}\right)$ (où $\left(\phi^{0}, z, \zeta\right) \in \mathcal{C U S}(\boldsymbol{G}, F)$ ) forment un système de représentants de l'ensemble des classes d'isomorphismes de paires cuspidales $F$-stables de $\boldsymbol{G}$.

On notera $A_{\phi^{0}, z, \zeta}$ le faisceau pervers sur $\boldsymbol{G}$ obtenu comme extension perverse (pour la perversité usuelle) du système local $\mathcal{E}_{\phi^{0}, z, \zeta}$ sur $z \boldsymbol{Z}^{0} . C_{\text {reg }}$. C'est un faisceau-caractère cuspidal sur $\boldsymbol{G}$ et tous les faisceaux-caractères cuspidaux sur $\boldsymbol{G}$ sont obtenus de cette manière ( $c f$. [L3, IV, prop. 18.5, (a) et III, 7.1.2, 7.1.3 et 7.1.4]). Puisque $p$ est bon pour $\boldsymbol{G}$, cette extension perverse est simplement, à un décalage près $\operatorname{de} \operatorname{dim} \boldsymbol{Z}^{0}+\operatorname{dim} C_{\text {reg }}$, l'extension par zéro de $\mathcal{E}_{\phi^{0}, z, \zeta}(c f$. [L3, IV, prop. 18.5, (b)]).

6.2. Fonctions caractéristiques. - Si $\boldsymbol{X}$ est une variété définie sur $\mathbb{F}_{q}$, d'endomorphisme de Frobenius $F: \boldsymbol{X} \rightarrow \boldsymbol{X}$, et si $\mathcal{F}$ est un système local $F$-stable, alors, à tout isomorphisme $\varphi: F^{*} \mathcal{F} \rightarrow \mathcal{F}$, on associe une fonction caractéristique $\chi_{\mathcal{F}, \varphi}$ définie comme suit :

$$
\begin{aligned}
\chi_{\mathcal{F}, \varphi}: \boldsymbol{X}^{F} & \longrightarrow \overline{\mathbb{Q}}_{\ell}, \\
x & \longmapsto \operatorname{Tr}\left(\varphi_{x}, \mathcal{F}_{x}\right) .
\end{aligned}
$$

Si $A$ est un faisceau pervers $F$-stable sur $\boldsymbol{X}$ et si $\varphi: F^{*} A \rightarrow A$ est un isomorphisme de faisceux pervers, on appellera fonction caractéristique de $A$ et on notera $\chi_{A, \varphi}$ la fonction définit comme suit :

$$
\begin{aligned}
\chi_{A, \varphi}: \boldsymbol{X}^{F} & \longrightarrow \overline{\mathbb{Q}}_{\ell}, \\
x & \longmapsto \sum_{i \in \mathbb{Z}}(-1)^{i} \operatorname{Tr}\left(\varphi_{x}, \mathcal{H}_{x}^{i} A\right)
\end{aligned}
$$

TOME $128-2000-\mathrm{N}^{\circ} 3$ 
où, pour tout $i \in \mathbb{Z}, \mathcal{H}_{x}^{i} A$ désigne la fibre en $x$ du $i$-ième faisceau de cohomologie de $A$.

Soit $\iota=\left(\phi^{0}, z, \zeta\right) \in \mathcal{C U S}(\boldsymbol{G}, F)$. Alors il existe un unique isomorphisme $\varphi_{\phi^{0}}: F^{*} \mathcal{S}_{\phi^{0}} \rightarrow \mathcal{S}_{\phi^{0}}$ dont la fonction caractéristique sur $Z^{0 F}$ soit égale à $\phi^{0}$. De même, on fixe une racine de $q$ dans $\overline{\mathbb{Q}}_{\ell}$ (que l'on notera $q^{\frac{1}{2}}$ ) : alors il existe un unique isomorphisme $\varphi_{\zeta}: F^{*} \mathcal{L}_{\zeta} \rightarrow \mathcal{L}_{\zeta}$ dont la fonction caractéristique a pour valeur $q^{\frac{1}{2} \operatorname{dim} C_{U_{0}}\left(u_{1}\right)}$ en $u_{1}$ (on rappelle que $u_{1}$ est un élément fixé de $C_{\text {reg }}^{F}$ ). On notera $\varphi_{\iota}$ l'isomorphisme $T(\dot{z})_{*}\left(\varphi_{\phi^{0}} \otimes \varphi_{\zeta}\right): F^{*} \mathcal{E}_{\iota} \rightarrow \mathcal{E}_{\iota}$. Cet isomorphisme induit un isomorphisme de faisceaux pervers sur $G$ que l'on notera toujours $\varphi_{\iota}: F^{*} A_{\iota} \rightarrow A_{\iota}$. Nous allons décrire explicitement la fonction caractéristique associée à cet isomorphisme.

Soit $c \in H^{1}(F, \boldsymbol{Z})$. On pose $u_{c}=\tilde{g}_{c} u_{1} \tilde{g}_{c}^{-1}$. Alors les $u_{c}\left(c \in H^{1}(F, \boldsymbol{Z})\right)$ forment un système de représentants de l'ensemble des classes de conjugaison d'éléments unipotents réguliers de $\boldsymbol{G}^{F}$. Soit $g \in \boldsymbol{G}^{F}$. On a alors :

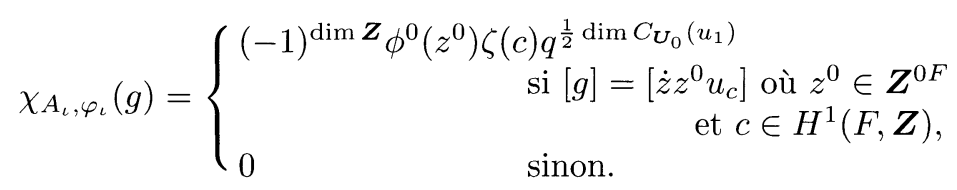

On choisit un caractère linéaire $\phi: \boldsymbol{Z}^{F} \rightarrow \overline{\mathbb{Q}}_{\ell}{ }^{\times}$dont la restriction à $\boldsymbol{Z}^{0 F}$ est égale à $\phi^{0}$ et on note $s$ l'unique (à $G^{* F^{*}}$-conjugaison près) élément semi-simple rationnellement cuspidal de $\boldsymbol{G}^{* F^{*}}$ tel que $\phi_{s}=\phi(c f$. th. 4.3.3, (a) et (b)). Soient $a \in A_{G^{*}}(s)^{F^{*}}$ et $\sigma \in H^{1}\left(F^{*}, A_{G^{*}}(s)\right)^{\wedge}$ tels que $\omega_{s}^{0}(a)=\zeta$ et $\widehat{\omega}_{s}^{1}(z)=\sigma$.

ThÉORÈme 6.2.2. - Avec les notations ci-dessus, il existe un racine de l'unité $\lambda \in \overline{\mathbb{Q}}_{\ell}{ }^{\times}$telle que

$$
\chi_{A_{\iota}, \varphi_{\iota}}=\lambda \widehat{\chi}_{s,(a, \sigma)}^{G} .
$$

Preuve. - D'après [B2, cor. 6.2.2], d'après 6.2.1 et d'après le théorème 4.3.4, il existe une constante $\lambda \in \overline{\mathbb{Q}}_{\ell}{ }^{\times}$satisfaisant à l'égalité du théorème 6.2.2. Il suffit de montrer que cette constante est une racine de l'unité. Pour cela, la valeur $\widehat{\chi}_{s,(a, \sigma)}^{\boldsymbol{G}}$ en $\dot{z} u_{1}$ permettra de conclure. Tout d'abord, $\widehat{\chi}_{s,(a, \sigma)}^{\boldsymbol{G}}\left(\dot{z} u_{1}\right)=\phi(\dot{z}) \widehat{\chi}_{s,(a, 1)}^{\boldsymbol{G}}\left(u_{1}\right)$. Cela montre que l'on peut supposer que $\dot{z}=1$ et donc que $\sigma=1$.

D'après le théorème 4.3.4, on a

$$
\begin{aligned}
\widehat{\chi}_{s,(a, 1)}^{\boldsymbol{G}} & =\sum_{(c, \xi) \in \mathcal{M}\left(\boldsymbol{Z} / \boldsymbol{Z}^{0}, F\right)} \frac{\zeta(c)}{\left|H^{1}(F, \boldsymbol{Z})\right|} \operatorname{Ind}_{\boldsymbol{Z}^{F} \times \boldsymbol{U}_{0}^{F}}^{\boldsymbol{G}^{F}}\left(\phi \xi \otimes \psi_{c}\right) \\
& =\sum_{c \in H^{1}(F, \boldsymbol{Z})} \frac{\zeta(c)}{\left|H^{1}(F, \boldsymbol{Z})\right|} \operatorname{Ind}_{\boldsymbol{Z}^{0 F} \times \boldsymbol{U}_{0}^{F}}^{\boldsymbol{G}^{F}}\left(\phi^{0} \otimes \psi_{c}\right) .
\end{aligned}
$$


Mais, si $c \in H^{1}(F, Z)$, on a

$$
\begin{aligned}
\operatorname{Ind}_{\boldsymbol{Z}^{0 F} \times \boldsymbol{U}_{0}^{F}}^{\boldsymbol{G}^{F}}\left(\phi^{0} \otimes \psi_{c}\right)\left(u_{1}\right) & =\frac{1}{\left|\boldsymbol{Z}^{0 F}\right| \cdot\left|\boldsymbol{U}_{0}^{F}\right|} \sum_{b \in \boldsymbol{B}_{0}^{F}} \psi_{c}\left(b u_{1} b^{-1}\right) \\
& =\sum_{t \in \boldsymbol{T}_{0}^{F} / \boldsymbol{Z}^{0 F}} \psi_{c}\left(t u_{1} t^{-1}\right)
\end{aligned}
$$

la première égalité résultant du fait que $\boldsymbol{B}_{0}$ est l'unique sous-groupe de Borel de $\boldsymbol{G}$ contenant $u_{1}$ car $u_{1}$ est régulier. Par conséquent,

$$
\widehat{\chi}_{s,(a, 1)}^{\boldsymbol{G}}\left(u_{1}\right)=\sum_{c \in H^{1}(F, \boldsymbol{Z})} \zeta(c)\left(\sum_{t \in \boldsymbol{T}_{0}^{F} / \boldsymbol{Z}^{F}} \psi_{c}\left(t u_{1} t^{-1}\right)\right) .
$$

Cette quantité est notée $\sigma_{\zeta}$ dans [DLM, partie 2]. En reprenant les notations de [DLM, partie 2], le sous-groupe de Levi noté $\boldsymbol{L}$ est égal à $\boldsymbol{G}$ car $\zeta$ est cuspidal et le résultat découle de [DLM, prop. 2.1] car $\operatorname{dim} \boldsymbol{G}-\operatorname{dim} C_{\mathrm{reg}}-\operatorname{dim} \boldsymbol{Z}=$ $\operatorname{dim} C_{\boldsymbol{U}_{0}}\left(u_{1}\right)$.

\section{BIBLIOGRAPHIE}

[A] AsAi (T.). - Twisting operators on the space of class functions of finite special linear groups, Proc. Symp. in Pure Math., t. 47, 1987, p. 99-148.

[B1] Bonnafé (C.). - Formule de Mackey pour q grand, J. Algebra, t. 201, 1998, p. 207-232.

[B2] Bonnafé (C.). - Mackey formula in type A, Proc. London Math. Soc., t. 80, 2000, p. 545-574.

[D] Digne (F.). - Descente de Shintani et descente des scalaires, J. London Math. Soc., t. 59, 1999, p. 867-880.

[DL] Deligne (P.), Lusztig(G.). - Representations of reductive groups over finite fields, Ann. of Math. (2), t. 103, 1976, p. 103-161.

[DLM] Digne (F.), Lehrer (G.), Michel (J.). - The characters of the group of rational points of a reductive group with non-connected centre, J. reine angew. Math., t. 425, 1992, p. 155-192.

[DM1] Digne (F.), Michel (J.). - Fonctions $\mathcal{L}$ des variétés de Deligne-Lusztig et descente de Shintani, Mém. Soc. Math. France, t. 20, 1985.

TOME $128-2000-\mathrm{N}^{\circ} 3$ 
[DM2] Digne (F.), Michel (J.). - On Lusztig's parametrization of characters of finite groups of Lie type, Astérisque 181-182, 1990, p. 113-156.

[DM3] Digne (F.), Michel (J.). - Representations of finite groups of Lie type. in London Math. Soc. Students Texts, vol. 21, Cambridge University Press, 1991.

[K1] Kawanaka (N.). - Generalized Gel'fand-Graev representations and Ennola duality, Adv. Stud. in Pure Math., Algebraic groups and related topics, t. 6, 1985, p. $175-206$.

[K2] Kawanaka (N.). - Generalized Gel'fand-Graev representations of exceptional simple algebraic groups over a finite field, I, Invent. Math., t. 84, 1986 , p. $575-616$.

[Lou] Lou (B.). - The centralizers of a regular unipotent element in a semisimple algebraic group, Bull. A.M.S., t. 74, 1968, p. 1144-1146.

[L1] Lusztig (G.). - Characters of reductive groups over finite fields, Ann. of Math. Studies, t. 107, 1984 .

[L2] Lusztig (G.). - Intersection cohomology complexes on reductive groups, Invent. Math., t. 75, 1984, p. 205-272.

[L3] Lusztig (G.).- Character sheaves, I, Adv. in Math., t. 56, 1985, p. 193-237; Character sheaves, II, t. 57, 1985, p. 226-265; Character sheaves, III, t. 57, 1985, p. 266-315; Character sheaves, IV, t. 59, 1986, p. 1-63; Character sheaves, V, t. 61, 1986, p. 103-155.

[LS] Lusztig (G.), SRinivasan (B.). - The characters of the finite unitary groups, J. Algebra, t. 49, 1977, p. 167-171.

[Sh1] Shoji (T.). - Shintani descent for algebraic groups over a finite field, I, J. Algebra, t. 145, 1992, p. 468-524.

[Sh2] Shour (T.). - Character sheaves and almost characters of reductive groups, I et II, Adv. in Math., t. 111, 1995, p. 244-313 et 314-354.

[Sh3] Shou (T.). - Shintani descent for special linear groups, J. Algebra, t. 199, 1998, p. $175-228$.

[Sp] Springer (T.A.). - Some arithmetic results on semi-simple Lie algebras, Publ. Math. I.H.E.S, t. 30, 1966, p. 115-141.

[SpSt] Springer (T.A.), Steinberg (R.). - Conjugacy classes, Seminar in algebraic groups and related topics, Lecture Notes in Math., t. 131, 1970, p. 167-266.

[St1] Steinberg (R.). - Regular elements of semisimple algebraic groups, Publ. Math. I.H.E.S., t. 25, 1965.

[St2] Steinberg (R.). - Endomorphisms of linear algebraic groups, Memoirs of the A.M.S., t. 80, 1968. 